\title{
Photon-assisted Andreev tunneling through a mesoscopic hybrid system
}

\author{
Qing-feng Sun \\ Department of Physics, The University of Hong Kong, Pokfulam Road, Hong Kong, China \\ and Department of Physics, Peking University, Beijing 100871, China \\ Jian Wang \\ Department of Physics, The University of Hong Kong, Pokfulam Road, Hong Kong, China \\ Tsung-han Lin* \\ Department of Physics, The University of Hong Kong, Pokfulam Road, Hong Kong, China \\ and Department of Physics, Peking University, Beijing 100871, China \\ (Received 6 July 1998; revised manuscript received 14 September 1998)
}

\begin{abstract}
The electron tunneling through a mesoscopic hybrid system, a normal-metal-quantum-dot-superconductor $(N-\mathrm{QD}-\mathrm{S})$ system where the intradot Coulomb interaction is neglected, in the presence of the time-varying external fields, has been investigated. By using the nonequilibrium Green-function method, the time-dependent current $j_{L}(t)$ and the average current $\langle j(t)\rangle$ are derived. The photon-assisted Andreev tunneling (PAAT) and the normal photon-assisted tunneling (PAT) are studied in detail. In the case of $\hbar \omega<\Delta$, where $\omega$ is the frequency of external fields and $\Delta$ is the energy gap of the superconducting lead, the average current $\langle j\rangle$ vs the gate voltage exhibits a series of equal-interval PAAT peaks, with negative peaks on the left-hand side and positive peaks on the right-hand side of the original resonant peak in the absence of the external fields. This is very different from the $N$-QD- $N$ system. While for $\hbar \omega>\Delta$, various PAT processes cause a rather complicated dependence of the current on the gate voltage. In addition, the current-bias-voltage characteristics become more complicated: each Andreev reflection peak is split into side-band peaks and each current plateau is split into substep plateaus. [S0163-1829(99)02819-2]
\end{abstract}

\section{INTRODUCTION}

Recently, the subject of time-dependent tunneling through a mesoscopic system has received more and more attention. The essential feature of mesoscopic physics is the phase coherence of the charge carriers. For time-dependent processes, generally, the external time-dependent perturbation affects the phase factor of the wave function differently in different regions of the system, ${ }^{1}$ leading to the well-known photonassisted tunneling (PAT), in which the electron can tunnel through the system by emitting or absorbing multiple photons. Such a PAT process is responsible for the side-band peaks in the curve of the conductance vs the gate voltage and the substep structure in the current-bias-voltage $(I-V)$ characteristics. $^{2,3}$

Experimentally, the PAT has been observed in a variety of systems, including single quantum dot, ${ }^{3-5}$ two-coupled quantum dots, ${ }^{6}$ and semiconductor superlattices, ${ }^{7}$ etc. Theoretically, Tien and Gordon studied the effect of microwave radiation on superconducting tunneling devices back to the early 1960's. ${ }^{8}$ Since then, different theoretical approaches have been developed, such as the time-dependent Schrödinger equation, ${ }^{9,10}$ the transfer Hamiltonian, ${ }^{11}$ the Master equation, ${ }^{12}$ the Wigner function, ${ }^{13}$ the nonequilibriumGreen-function (NGF) method, ${ }^{1,14,15}$ the Keldysh diagram technique, ${ }^{16}$ and the scattering matrix approach. ${ }^{17}$

In recent years, another subject that has been investigated extensively is the mesoscopic "hybrid" normal-metalsuperconductor systems. The interplay between basic features originated from both of mesoscopics and superconduc- tivity makes this subbranch of the condensed-matter physics a very fruitful research field. ${ }^{18-20}$ Many interesting phenomena have been studied for various mesoscopic "hybrid" systems, such as the maximum supercurrent quantization and nonsinusoidal behavior of the current-phase relation of the superconducting quantum point contacts, ${ }^{21-24}$ the subharmonic gap structure in $S-I-S$ or $S-N-S$ junctions, ${ }^{25}$ the Andreev-reflected bound states in $S-N-S$ or $N-I-N-S$ systems, ${ }^{26}$ the even-odd parity asymmetry and the Coulomb blockade of the Andreev reflection in superconductorsuperconducting-quantum-dot-superconductor ( $S$-SQD- $S$ ) or $N$-SQD- $N$ systems, ${ }^{27,28}$ etc.

For mesoscopic "hybrid" systems in the presence of external time-dependent fields, the situation becomes more complicated. Hergenrother et al. investigated the photonassisted tunneling through a single-electron tunneling transistor with a superconducting island, and observed that a striking secondary peak and a nonzero background current appear in the curve of the current $I$ vs the gate-induced charge $Q .{ }^{29}$ Antonov and Takayanagi measured the phasecoherent effect in the resistance of mesoscopic normalsuperconductor structures exposed to rf radiation. ${ }^{30}$ Hanke, Gisselfält, and Chao studied the photon-assisted Andreev tunneling (PAAT) through a normal-metal-superconductornormal-metal single-electron tunneling transistor with an oscillating potential coupled to the superconductor. ${ }^{31}$ Zhao et al. investigated the PAT through a normal-metal-normalquantum-dot-superconductor system without considering the PAAT process. $^{32}$

In this paper, we shall investigate the PAAT through a 
mesoscopic hybrid system, a normal-metal-quantum-dotsuperconductor system $(N-\mathrm{QD}-S)$, with time-dependent external fields. In the absence of external fields, this system has been studied before. Beenakker presented a general multichannel $S$-matrix description and predicted the resonant Andreev tunneling for a single-level QD in the zero-bias limit. ${ }^{33}$ Later, Claughton, Leadbeater, and Lambert extended this theory to the finite-bias case and found that differential conductance resonances are strongly suppressed in the weakly coupling limit. ${ }^{34}$ Recently, Fazio and Raimondi investigated how the Kondo effect can influence the two-particle tunneling through the $N$-QD- $S$ system with a strongly interacting QD. ${ }^{35}$

In this paper, we only consider a single energy level (but including the spin) in the quantum dot for simplicity. By using the nonequilibrium-Green-function (NGF) method, the time-dependent current $j_{L}(t)$, and the average current $\langle j\rangle$ are derived. Without the superconducting lead, i.e., for a $N$-QD- $N$ system, it has been studied experimentally and theoretically. It is found that when the external field is applied only on the right lead, the curve of the current $\langle j\rangle$ vs the gate voltage $v_{g}$ has a shoulder on the left-hand side of the original resonant peak, and a negative current on the righthand side. ${ }^{2,4}$ When the external field is applied only on the quantum dot, the original resonant peak will be split into a series of side-band peaks with equal spacing $\hbar \omega$ due to the PAT (Refs. 2, 3, 9, and 15), where $\omega$ is the frequency of the external time-dependent fields. Now for the $N$-QD- $S$ system under consideration, in addition to the normal PAT, an interesting process, the PAAT, occurs. For $\hbar \omega<\Delta$, where $\Delta$ is the energy gap of the superconducting lead, and at the small dc bias, the PAAT is the dominate process in the tunneling. We find the following: (1) $A^{\prime}$ series of peaks with the equal interval $\frac{1}{2} \hbar \omega$ will emerge, due to the PAAT in which two electrons in the dot can tunnel into the right superconducting electrode, form a Cooper pair, at the same time, absorbing or emitting $n$ photon. (2) A series of negative peaks appear on the left-hand side and a series of positive peaks on the righthand side of the original resonant peak, respectively, in the absence of external fields. (3) Because the existence of the energy gap in the superconducting lead and its property of the density of states, the normal PAT processes become more complicated, leading to a rather complex pattern of the current $\langle j\rangle$ vs the gate voltage $v_{g}$. (4) In the current-biasvoltage characteristics, the original Andreev-reflection peaks and the conventional current plateaus will be split into a series of side-band peaks and the substep plateaus, respectively.

The rest of this paper is organized as follows. In Sec. II, the model is presented and the formulas of the timedependent current $j(t)$ and the average current $\langle j\rangle$ are derived using NGF technique. In Sec. III, we study the properties of the average current $\langle j\rangle$ vs the gate voltage $v_{g}$ while the timedependent external fields with equal amplitudes are applied on the quantum dot and the left lead, i.e., only the electron tunneling through the right barrier can absorb or emit photons. Specifically, we investigate two cases of $\hbar \omega<\Delta$ and $\hbar \omega>\Delta$ in detail. In Sec. IV, we study the the properties of $\langle j\rangle$ vs $v_{g}$ when the time-dependent external field is applied only in the quantum dot. The properties of the current $\langle j\rangle$ vs the dc bias $V$ are presented in Sec. V. Finally, a brief summary is given in Sec. VI.

\section{MODEL AND FORMULATION}

We assume that the system under consideration is described by the following Hamiltonian $H(t):{ }^{23,36}$

$$
H(t)=H_{L}(t)+H_{\mathrm{dot}}(t)+H_{R}+H_{T},
$$

where

$$
\begin{aligned}
& H_{L}(t)=\sum_{k, \sigma} \epsilon_{k}(t) a_{k \sigma}^{\dagger} a_{k \sigma}, \\
& H_{\mathrm{dot}}(t)=\sum_{\sigma} \epsilon_{d \sigma}(t) c_{\sigma}^{\dagger} c_{\sigma},
\end{aligned}
$$

$$
H_{R}=\sum_{p, \sigma} \epsilon_{p} b_{p \sigma}^{\dagger} b_{p \sigma}+\sum_{p}\left[\Delta^{*} b_{p \downarrow} b_{-p \uparrow}+\Delta b_{-p \uparrow}^{\dagger} b_{p \downarrow}^{\dagger}\right],
$$

$H_{T}=\sum_{k, \sigma}\left[L_{k} a_{k \sigma}^{\dagger} c_{\sigma}+L_{k}^{*} c_{\sigma}^{\dagger} a_{k \sigma}\right]+\sum_{p, \sigma}\left[R_{p} b_{p \sigma}^{\dagger} c_{\sigma}+R_{p}^{*} c_{\sigma}^{\dagger} b_{p \sigma}\right]$.

$H_{L}(t)$ describes noninteracting electrons in the left normalmetal lead, $a_{k \sigma}^{\dagger}\left(a_{k \sigma}\right)$ are the creation (annihilation) operators of the electron in the left lead. $H_{\mathrm{dot}}(t)$ models the quantum dot with single level but including spin. For simplicity, the intradot electron-electron Coulomb interaction is not considered, namely, we only consider a big dot. $H_{R}$ describes the right superconducting lead with the energy gap $\Delta$. Here, we have set the chemical potential of the right lead to be zero due to the gauge invariance in the following sense: if the voltages of the left lead, the right lead, and the gate are all shifted by the same amount, the average current does not change. ${ }^{37} \mathrm{We}$ assume that the external time-dependent fields are only applied on the left lead and the quantum dot, but not on the right superconducting lead, so $H_{R}$ is time independent. In fact, we have proved that the case with external fields applied to all three regions (the left lead, the dot, and the right superconducting lead) is exactly equivalent to the case with the external fields only applied on the left lead and the dot, but not on the right lead (see Appendix A). We also assume that the frequency of the external fields is not too high so that the adiabatic approximation holds. ${ }^{1,15,38}$ According to the estimation by Wingreen and co-workers, ${ }^{1}$ the upper limit of the frequency is the plasma frequency, about tens of $\mathrm{THz}$ for typical doped semiconductor samples, and higher for metallic materials. Under the adiabatic approximation, the time-dependent external fields are contained in the single-electron energies $\epsilon_{\alpha \sigma}(t)$ (where $\alpha=k$ or $d$ corresponds to the left lead or the dot, respectively), and the distributions of the electrons in the left lead remain unchanged. We separate $\epsilon_{\alpha \sigma}(t)$ into two parts: $\epsilon_{\alpha \sigma}(t)=\epsilon_{\alpha \sigma}+W_{\beta}(t)$, where $\beta=L, d$ corresponds to the left lead and the dot, respectively; $\epsilon_{\alpha \sigma}$ is the time-independent single-electron energies without the time-dependent external fields, and $W_{\beta}(t)$ is a time-dependent part from the external fields. ${ }^{1,15,38} H_{T}$ denotes the tunneling part, which is time-independent.

The current from the left normal-metal lead flowing into the quantum dot can be calculated from the evolution of the 
total number operator of the electrons in the left lead, $N_{L}$ $=\Sigma_{k, \sigma} a_{k \sigma}^{\dagger} a_{k \sigma}:{ }^{1,15}$ (in units of $\hbar=1$ )

$$
\begin{aligned}
j_{L}(t)= & -e\left\langle\dot{N}_{L}(t)\right\rangle=i e\left\langle\left[N_{L}, H\right]\right\rangle \\
= & -2 e \sum_{\sigma} \operatorname{Im} \int_{-\infty}^{t} d t_{1} \int \frac{d \epsilon}{2 \pi} \Gamma_{L}(\epsilon)\left[G_{\sigma \sigma}^{<}\left(t, t_{1}\right)\right. \\
& \left.+f_{L}(\epsilon) G_{\sigma \sigma}^{r}\left(t, t_{1}\right)\right] \exp \left(-i \epsilon\left(t_{1}-t\right)-i \int_{t}^{t_{1}} W_{L}(\tau) d \tau\right) \\
\equiv & j_{L \uparrow}(t)+j_{L \downarrow}(t),
\end{aligned}
$$

where we define the Green's functions: $G_{\sigma \sigma}^{<}\left(t, t_{1}\right) \equiv i\left\langle c_{\sigma}^{\dagger}\left(t_{1}\right) c_{\sigma}(t)\right\rangle, \quad$ and $\quad G_{\sigma \sigma}^{r}\left(t, t_{1}\right) \equiv-i \theta(t$ $\left.-t_{1}\right)\left\langle\left\{c_{\sigma}(t), c_{\sigma}^{\dagger}\left(t_{1}\right)\right\}\right\rangle . \quad f_{L(R)}(\epsilon)=\left\{\exp \left[\left(\epsilon-\mu_{L(R)}\right) / k_{B} \mathcal{T}\right]\right.$ $+1\}^{-1}$ is the Fermi-distribution function of the left (right) lead where $\mu_{L}=e V$ and $\mu_{R}=0$. Here, $\Gamma_{L}(\epsilon)$ is the linewidth function defined by $\Gamma_{L}(\epsilon) \equiv 2 \pi \rho_{L}(\epsilon) L(\epsilon) L^{*}(\epsilon)$, where $\rho_{L}(\epsilon)$ is the density of states in the left lead, and $L\left(\epsilon_{k}\right)$ $=L_{k}$.

In the following, we derive the expression of the timedependent current $j_{L \uparrow}(t)$. From $j_{L \uparrow}(t)$, the current $j_{L \downarrow}(t)$ is easily obtained by exchange the spin index. Because the right lead is in superconducting state, it is convenient to use the $2 \times 2$ Nambu representation in which $\mathbf{G}^{<}\left(t, t^{\prime}\right)$ and $\mathbf{G}^{r}\left(t, t^{\prime}\right)$ take the forms ${ }^{23}$

$$
\mathbf{G}^{<}\left(t, t^{\prime}\right) \equiv i\left(\begin{array}{ll}
\left\langle c_{\uparrow}^{\dagger}\left(t^{\prime}\right) c_{\uparrow}(t)\right\rangle & \left\langle c_{\downarrow}\left(t^{\prime}\right) c_{\uparrow}(t)\right\rangle \\
\left\langle c_{\uparrow}^{\dagger}\left(t^{\prime}\right) c_{\downarrow}^{\dagger}(t)\right\rangle & \left\langle c_{\downarrow}\left(t^{\prime}\right) c_{\downarrow}^{\dagger}(t)\right\rangle
\end{array}\right),
$$

$$
\begin{aligned}
\mathbf{G}^{r}\left(t, t^{\prime}\right) \equiv & -i \theta\left(t-t^{\prime}\right) \\
& \times\left(\begin{array}{ll}
\left\langle\left\{c_{\uparrow}(t), c_{\uparrow}^{\dagger}\left(t^{\prime}\right)\right\}\right\rangle & \left\langle\left\{c_{\uparrow}(t), c_{\downarrow}\left(t^{\prime}\right)\right\}\right\rangle \\
\left\langle\left\{c_{\downarrow}^{\dagger}(t), c_{\uparrow}^{\dagger}\left(t^{\prime}\right)\right\}\right\rangle & \left\langle\left\{c_{\downarrow}^{\dagger}(t), c_{\downarrow}\left(t^{\prime}\right)\right\}\right\rangle
\end{array}\right) .
\end{aligned}
$$

In terms of Green's functions $\mathbf{G}^{<}\left(t, t^{\prime}\right)$ and $\mathbf{G}^{r}\left(t, t^{\prime}\right)$, the current $j_{L \uparrow}(t)$ is given by

$$
\begin{aligned}
j_{L \uparrow}(t)= & -2 e \operatorname{Im} \int_{-\infty}^{t} d t^{\prime} \int \frac{d \epsilon}{2 \pi} \Gamma_{L}(\epsilon) \exp \left(-i \epsilon\left(t^{\prime}-t\right)\right. \\
& \left.-i \int_{t}^{t^{\prime}} W_{L}(\tau) d \tau\right)\left[\mathbf{G}^{<}\left(t, t^{\prime}\right)+f_{L}(\boldsymbol{\epsilon}) \mathbf{G}^{r}\left(t, t^{\prime}\right)\right]_{11}
\end{aligned}
$$

In order to obtain the expression of the current $j_{L \uparrow}(t)$, we have to solve the Green's functions $\mathbf{G}^{<}\left(t, t^{\prime}\right)_{11}$ and $\mathbf{G}^{r}\left(t, t^{\prime}\right)_{11}$. $\quad \mathbf{G}^{<}\left(t, t^{\prime}\right)_{11}$ is related to $\mathbf{G}^{r}\left(t, t^{\prime}\right)$ through the Keldysh equation

$$
\mathbf{G}^{<}\left(t, t^{\prime}\right)=\iint d t_{1} d t_{2} \mathbf{G}^{r}\left(t, t_{1}\right) \Sigma^{<}\left(t_{1}, t_{2}\right) \mathbf{G}^{a}\left(t_{2}, t^{\prime}\right),
$$

where $\Sigma^{<}\left(t_{1}, t_{2}\right)$ is the self-energy. Here, we take the widebandwidth approximation, under that the linewidth $\Gamma_{L}\left(\Gamma_{R}\right)$ is independent with the energy $\epsilon$. Notice that $\Gamma_{R}$ $\equiv 2 \pi R(\epsilon) R^{*}(\epsilon) \rho_{R}^{N}(\epsilon)$, where $\rho_{R}^{N}(\epsilon)$ is the density of states in the right lead when it is in normal state. So here, the wide-bandwidth approximation is generally reasonable as for the normal system. ${ }^{15,38}$ In a normal mesoscopic system, the wide-bandwidth approximation (WBA) has been widely used. WBA is justified under the following conditions: (i) the bandwidth of the leads is much larger than the linewidth $\Gamma(\epsilon)$; (ii) the density of states $\left[\rho_{\alpha}(\epsilon)(\alpha=L, R)\right]$ and the hopping matrix elements $\left(L_{k}\right.$ and $\left.R_{p}\right)$ vary slowly with energy over a range of several $\Gamma$ around the resonant level, $\epsilon_{d \sigma}$; (iii) the energy level of the quantum dot $\epsilon_{d \sigma}$ is not close to the band bottom of the leads. Under the wide-bandwidth approximation, the self-energy $\Sigma^{<}\left(t_{1}, t_{2}\right)$ becomes

$$
\Sigma^{<}\left(t_{1}, t_{2}\right)=\Sigma_{L}^{<}\left(t_{1}, t_{2}\right)+\Sigma_{R}^{<}\left(t_{1}, t_{2}\right)
$$

where

$$
\begin{aligned}
& \Sigma_{L}^{<}\left(t_{1}, t_{2}\right)=\sum_{k} \mathbf{L}_{k} \mathbf{g}_{k}^{<}\left(t_{1}, t_{2}\right) \mathbf{L}_{k}^{*} \\
& =i \Gamma_{L} \int \frac{d \epsilon}{2 \pi}\left(\begin{array}{cc}
\exp \left(-i \epsilon\left(t_{1}-t_{2}\right)-i \int_{t_{2}}^{t_{1}} W_{L}(\tau) d \tau\right) f_{L}(\epsilon) & 0 \\
0 & \exp \left(i \epsilon\left(t_{1}-t_{2}\right)+i \int_{t_{2}}^{t_{1}} W_{L}(\tau) d \tau\right)\left[1-f_{L}(\epsilon)\right]
\end{array}\right),
\end{aligned}
$$


and $\Sigma_{R}^{<}\left(t_{1}, t_{2}\right)$ is given by (see Appendix B)

$$
\begin{aligned}
\Sigma_{R}^{<}\left(t_{1}, t_{2}\right)= & \sum_{p} \mathbf{R}_{p} \mathbf{g}_{p}^{<}\left(t_{1}, t_{2}\right) \mathbf{R}_{p}^{*} \\
= & i \Gamma_{R} \int \frac{d \boldsymbol{\epsilon}}{2 \pi} e^{-i \epsilon\left(t_{1}-t_{2}\right)} f_{R}(\boldsymbol{\epsilon}) \widetilde{\rho}_{R}(\boldsymbol{\epsilon}) \\
& \times\left(\begin{array}{cc}
1 & -\Delta /|\omega| \\
-\Delta /|\omega| & 1
\end{array}\right) .
\end{aligned}
$$

$\mathbf{L}_{k}$ and $\mathbf{R}_{p}$ in Eqs. (8) and (9) are the $2 \times 2$ matrix of the hopping elements

$$
\mathbf{L}_{k}\left(\mathbf{R}_{p}\right)=\left[\begin{array}{cc}
L_{k}\left(R_{p}\right) & 0 \\
0 & -L_{k}^{*}\left(-R_{p}^{*}\right)
\end{array}\right] .
$$

In Eqs. (8) and (9), $\mathbf{g}_{k(p)}^{<}\left(t_{1}, t_{2}\right)$ is the Green's function of the electron in the left (right) lead. $\widetilde{\rho}_{R}(\epsilon)$ in Eq. (9) is the corresponding dimensionless BCS density of states, i.e., $\widetilde{\rho}_{R}(\epsilon)$ is the ratio of the superconducting density of states $\rho_{R}^{S}(\epsilon)$ to the normal density of states $\rho_{R}^{N}(\epsilon): \tilde{\rho}_{R}(\epsilon)=\rho_{R}^{S}(\epsilon) / \rho_{R}^{N}(\epsilon)$. From $\rho_{R}^{S}(\epsilon)=(1 / \pi) i \operatorname{Im} \Sigma_{p} g_{p}^{a}(\epsilon)$, one easily finds ${ }^{23}$

$$
\widetilde{\rho}_{R}(\epsilon)=\theta(|\epsilon|-\Delta)|\epsilon| / \sqrt{\epsilon^{2}-\Delta^{2}} .
$$

Since the current $j_{L \uparrow}(t)$ only depends on $\left|R_{p}\right|^{2}$ and $\left|L_{k}\right|^{2}$, we have assumed for simplicity that $L_{k}$ and $R_{p}$ are real.

Substituting the expression of the self-energy $\Sigma^{<}\left(t_{1}, t_{2}\right)$, Eqs. (8) and (9), into the Keldysh equation, Eq. (7), we obtain

$$
\begin{aligned}
\mathbf{G}^{<}(t, t)_{11}= & \int \frac{d \boldsymbol{\epsilon}}{2 \pi}\left\{i \Gamma_{L} f_{L}(\boldsymbol{\epsilon})\left|\mathbf{A}_{L}(\boldsymbol{\epsilon}, t)_{11}\right|^{2}\right. \\
& \left.+i \Gamma_{L}\left[1-f_{L}(-\boldsymbol{\epsilon})\right]\left|\mathbf{B}_{L}(\boldsymbol{\epsilon}, t)_{12}\right|^{2}\right\} \\
& +\int \frac{d \boldsymbol{\epsilon}}{2 \pi} i \Gamma_{R} \widetilde{\rho}_{R}(\boldsymbol{\epsilon}) f_{R}(\boldsymbol{\epsilon}) \\
& \times\left\{\left|\mathbf{A}_{R}(\boldsymbol{\epsilon}, t)_{11}\right|^{2}+\left|\mathbf{A}_{R}(\boldsymbol{\epsilon}, t)_{12}\right|^{2}\right. \\
& \left.-\frac{2 \Delta}{|\boldsymbol{\epsilon}|} \operatorname{Re}\left[\mathbf{A}_{R}(\boldsymbol{\epsilon}, t)_{11} \mathbf{A}_{R}^{*}(\boldsymbol{\epsilon}, t)_{12}\right]\right\} .
\end{aligned}
$$

Here $\mathbf{A}_{L}(\boldsymbol{\epsilon}, t), \mathbf{A}_{R}(\boldsymbol{\epsilon}, t)$, and $\mathbf{B}_{L}(\boldsymbol{\epsilon}, t)$ are defined as

$$
\begin{gathered}
\mathbf{A}_{L}(\epsilon, t)=\int d t_{1} \mathbf{G}^{r}\left(t, t_{1}\right) \exp \left(-i \epsilon\left(t_{1}-t\right)-i \int_{t}^{t_{1}} W_{L}(\tau) d \tau\right), \\
\mathbf{A}_{R}(\epsilon, t)=\int d t_{1} \mathbf{G}^{r}\left(t, t_{1}\right) e^{-i \epsilon\left(t_{1}-t\right)}, \\
\mathbf{B}_{L}(\boldsymbol{\epsilon}, t)=\int d t_{1} \mathbf{G}^{r}\left(t, t_{1}\right) \exp \left(-i \epsilon\left(t_{1}-t\right)+i \int_{t}^{t_{1}} W_{L}(\tau) d \tau\right) .
\end{gathered}
$$

Substituting the Green's function $\mathbf{G}^{<}(t, t)_{11}$ into Eq. (6), the time-dependent current $j_{L \uparrow}(t)$ can be expressed as

$$
\begin{aligned}
j_{L \uparrow}(t)= & -e \int \frac{d \boldsymbol{\epsilon}}{2 \pi} f_{L}(\boldsymbol{\epsilon})\left\{\Gamma_{L}^{2}\left|\mathbf{A}_{L}(\boldsymbol{\epsilon}, t)_{11}\right|^{2}\right. \\
& \left.+2 \Gamma_{L} \operatorname{Im} \mathbf{A}_{L}(\boldsymbol{\epsilon}, t)_{11}\right\} \\
& -e \int \frac{d \boldsymbol{\epsilon}}{2 \pi}\left[1-f_{L}(-\boldsymbol{\epsilon})\right] \Gamma_{L}^{2}\left|\mathbf{B}_{L}(\boldsymbol{\epsilon}, t)_{12}\right|^{2} \\
& -e \int \frac{d \boldsymbol{\epsilon}}{2 \pi} f_{R}(\boldsymbol{\epsilon}) \Gamma_{L} \Gamma_{R} \widetilde{\rho}_{R}(\boldsymbol{\epsilon}) \\
& \times\left\{\left|\mathbf{A}_{R}(\boldsymbol{\epsilon}, t)_{11}\right|^{2}+\left|\mathbf{A}_{R}(\boldsymbol{\epsilon}, t)_{12}\right|^{2}\right. \\
& \left.-\frac{2 \Delta}{|\epsilon|} \operatorname{Re}\left[\mathbf{A}_{R}(\boldsymbol{\epsilon}, t)_{11} \mathbf{A}_{R}^{*}(\boldsymbol{\epsilon}, t)_{12}\right]\right\}
\end{aligned}
$$

Next, we need to solve Green's functions $\mathbf{G}^{r}\left(t, t^{\prime}\right)_{11}$ and $\mathbf{G}^{r}\left(t, t^{\prime}\right)_{12}$. From the Dyson's equation, we have

$$
\begin{aligned}
\mathbf{G}^{r}\left(t, t^{\prime}\right)_{11}= & \mathbf{g}^{r}\left(t, t^{\prime}\right)_{11} \\
& +\iint d t_{1} d t_{2} \mathbf{G}^{r}\left(t, t_{1}\right)_{11} \Sigma^{r}\left(t_{1}, t_{2}\right)_{11} \mathbf{g}^{r}\left(t_{2}, t^{\prime}\right)_{11} \\
& +\iint d t_{1} d t_{2} \mathbf{G}^{r}\left(t, t_{1}\right)_{12} \Sigma^{r}\left(t_{1}, t_{2}\right)_{21} \mathbf{g}^{r}\left(t_{2}, t^{\prime}\right)_{11},
\end{aligned}
$$

$$
\begin{aligned}
\mathbf{G}^{r}\left(t, t^{\prime}\right)_{12}= & \iint d t_{1} d t_{2} \mathbf{G}^{r}\left(t, t_{1}\right)_{11} \Sigma^{r}\left(t_{1}, t_{2}\right)_{12} \mathbf{g}^{r}\left(t_{2}, t^{\prime}\right)_{22} \\
& +\iint d t_{1} d t_{2} \mathbf{G}^{r}\left(t, t_{1}\right)_{12} \Sigma^{r}\left(t_{1}, t_{2}\right)_{22} \mathbf{g}^{r}\left(t_{2}, t^{\prime}\right)_{22},
\end{aligned}
$$

where $\Sigma^{r}\left(t_{1}, t_{2}\right)$ is the self-energy. In the wide-bandwidth approximation, ${ }^{15,38}$ the self-energy $\Sigma^{r}\left(t_{1}, t_{2}\right)$ can be obtained as (see Appendix B) $\Sigma^{r}\left(t_{1}, t_{2}\right)=\Sigma_{L}^{r}\left(t_{1}, t_{2}\right)+\Sigma_{R}^{r}\left(t_{1}, t_{2}\right)$, with

$$
\Sigma_{L}^{r}\left(t_{1}, t_{2}\right)=\sum_{k} \mathbf{L}_{k} \mathbf{g}_{k}^{r}\left(t_{1}, t_{2}\right) \mathbf{L}_{k}^{*}=-\frac{i \Gamma_{L}}{2} \delta\left(t_{1}-t_{2}\right)\left(\begin{array}{ll}
1 & 0 \\
0 & 1
\end{array}\right),
$$

$$
\begin{aligned}
\sum_{R}^{r}\left(t_{1}, t_{2}\right) & =\sum_{p} \mathbf{R}_{p} \mathbf{g}_{p}^{r}\left(t_{1}, t_{2}\right) \mathbf{R}_{p}^{*} \\
& =-i \Gamma_{R} \int \frac{d \epsilon}{2 \pi} \frac{\theta\left(t_{1}-t_{2}\right) e^{-i \epsilon\left(t_{1}-t_{2}\right)}}{\sqrt{\epsilon^{2}-\Delta^{2}}}\left(\begin{array}{cc}
|\epsilon| & -\Delta \\
-\Delta & |\epsilon|
\end{array}\right)
\end{aligned}
$$

In Eqs. (15) and (16), $\mathbf{g}^{r}\left(t_{1}, t_{2}\right)$ is the Green's function of the electron in the dot without the coupling between the dot and two leads, and can be easily obtained as 


$$
\mathbf{g}^{r}\left(t_{1}, t_{2}\right)=-i \theta\left(t_{1}-t_{2}\right)\left(\begin{array}{cc}
\exp \left(-i \int_{t_{2}}^{t_{1}} \epsilon_{d \uparrow}(\tau) d \tau\right) & 0 \\
0 & \exp \left(i \int_{t_{2}}^{t_{1}} \epsilon_{d \downarrow}(\tau) d \tau\right)
\end{array}\right) .
$$

Notice that these Green's functions of the dot depend on two time variables, not the time difference; therefore one should take the Fourier expansion of the Green's functions as ${ }^{23}$

$$
G\left(t, t_{1}\right)=\sum_{n} e^{i n \omega t_{1}} \int \frac{d \epsilon}{2 \pi} e^{-i \epsilon\left(t-t_{1}\right)} G_{n}(\epsilon) .
$$

Here, $\omega$ is the frequency of external fields. To simplify the discussion, in the following we only consider the harmonic external field, i.e., $W_{\beta}(t)=W_{\beta} \cos \omega t(\beta=L, d){ }^{1,2}$ Introducing the notation $G_{m n}(\epsilon) \equiv G_{n-m}(\epsilon+m \omega),{ }^{23}$ [here and in Eq. (20), $G$ can be $g^{r}, \Sigma_{R}^{r}$, etc.] noticing that different components $G_{m n}$ are related by $G_{m n}(\epsilon)=G_{0, n-m}(\epsilon+m \omega)$, then the Fourier transformation of the Green's function $\mathbf{g}^{r}\left(t, t^{\prime}\right)$ and the self-energy $\Sigma^{r}\left(t, t^{\prime}\right)$ are easily obtained

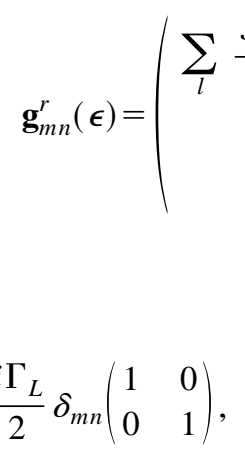

$$
\begin{aligned}
& \Sigma_{R, m n}^{r}(\epsilon)=-\frac{i \Gamma_{R}}{2} \frac{\delta_{m n}}{\sqrt{\left(\epsilon+m \omega+i 0^{+}\right)^{2}-\Delta^{2}}} \\
& \times\left(\begin{array}{cc}
\left|\epsilon+m \omega+i 0^{+}\right| & -\Delta \\
-\Delta & \left|\epsilon+m \omega+i 0^{+}\right|
\end{array}\right),
\end{aligned}
$$

where $\alpha_{R}=W_{d} / \omega$. And $\mathbf{g}_{m n}^{r}, \Sigma_{L, m n}^{r}$, and $\Sigma_{R, m n}^{r}$ are defined in Eq. (20). Notice that the self-energy $\Sigma_{R}^{r}\left(t, t^{\prime}\right)$ depends only on the time difference, so $\Sigma_{R, m n}^{r}(\epsilon)$ is zero for $m \neq n$. After taking the Fourier transformations, Eqs. (15) and (16) become

$$
\begin{aligned}
G_{m n ; 11}^{r}= & g_{m n ; 11}^{r}+\sum_{m_{1}} G_{m m_{1} ; 11}^{r} \Sigma_{m_{1} m_{1} ; 11}^{r} g_{m_{1} n ; 11}^{r} \\
& +\sum_{m_{1}} G_{m m_{1} ; 12}^{r} \Sigma_{m_{1} m_{1} ; 21}^{r} g_{m_{1} n ; 11}^{r} \\
G_{m n ; 12}^{r}= & \sum_{m_{1}} G_{m m_{1} ; 11}^{r} \Sigma_{m_{1} m_{1} ; 12}^{r} g_{m_{1} n ; 22}^{r} \\
& +\sum_{m_{1}} G_{m m_{1} ; 12}^{r} \Sigma_{m_{1} m_{1} ; 22}^{r} g_{m_{1} n ; 22}^{r}
\end{aligned}
$$

where we have suppressed the argument $\epsilon$. By iterating Eq. (25), then substituting its formal solution $G_{m n ; 12}^{r}$ into Eq. (24), one easily finds

$$
\begin{aligned}
G_{m n ; 11}^{r}= & g_{m n ; 11}^{r}+\sum_{m_{1}} G_{m m_{1} ; 11}^{r} \Sigma_{m_{1} m_{1} ; 11}^{r} g_{m_{1} n ; 11}^{r} \\
& +\sum_{m_{1}, m_{2}} G_{m m_{1} ; 11}^{r} \Sigma_{m_{1} m_{1} ; 12}^{r} D_{m_{1} m_{2}} \Sigma_{m_{2} m_{2} ; 21}^{r} g_{m_{2} n ; 11}^{r},
\end{aligned}
$$

where $D_{m n}$ is defined by

$$
D_{m n}=g_{m n ; 22}^{r}+\sum_{m_{1}} g_{m m_{1} ; 22}^{r} \Sigma_{m_{1} m_{1} ; 22}^{r} g_{m_{1} n ; 22}^{r}+\cdots .
$$

Substituting the Green's function $g_{m n ; 22}^{r}$, Eq. (21), and the self-energy function $\Sigma_{m m ; 22}^{r}$, Eqs. (22) and (23), into Eq. (27), $D_{m n}(\epsilon)$ can be obtained as

$$
D_{m n}=\sum_{l} \frac{J_{l-m}\left(\alpha_{R}\right) J_{l-n}\left(\alpha_{R}\right)}{\epsilon+\epsilon_{d \downarrow}+l \omega-\widetilde{\Sigma}_{l}},
$$

where

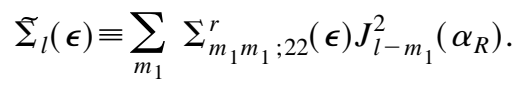

In deriving Eq. (28), we have taken the approximation

$$
\begin{aligned}
& \sum_{l, l^{\prime}} \frac{f_{l l^{\prime}}}{\left(\epsilon+\epsilon_{d \downarrow}+l \omega+i 0^{+}\right)\left(\epsilon+\epsilon_{d \downarrow}+l^{\prime} \omega+i 0^{+}\right)} \\
& \quad \approx \sum_{l} \frac{f_{l l}}{\left(\epsilon+\epsilon_{d \downarrow}+l \omega+i 0^{+}\right)^{2}},
\end{aligned}
$$

where $f_{l l^{\prime}}$ can be any function. This approximation is reasonable for $\omega \gg \Gamma\left(\Gamma \equiv \Gamma_{L}+\Gamma_{R}\right)$. Substituting $D_{m n}(\epsilon)$, $\mathbf{g}_{m n}^{r}(\epsilon)$, and $\Sigma_{m n}^{r}$, into Eq. (26), the Green's function $G_{m n, 11}^{r}(\epsilon)$ is obtained 


$$
G_{m n ; 11}^{r}=\sum_{l} J_{l+m}\left(\alpha_{R}\right) J_{l+n}\left(\alpha_{R}\right) \widetilde{G}_{l}(\epsilon)
$$

where

$$
\begin{aligned}
\widetilde{G}_{l}(\epsilon)= & {\left[\epsilon-\epsilon_{d \uparrow}-l \omega-\sum_{m_{1}} J_{l+m_{1}}^{2}\left(\alpha_{R}\right) \Sigma_{m_{1} m_{1} ; 11}^{r}\right.} \\
& \left.-\sum_{l^{\prime}} \frac{\left[\widetilde{\Sigma}_{l l^{\prime}}(\epsilon)\right]^{2}}{\epsilon+\epsilon_{d \downarrow}+l^{\prime} \omega-\widetilde{\Sigma}_{l^{\prime}}}\right]^{-1},
\end{aligned}
$$

with

$$
\Sigma_{l l^{\prime}}(\epsilon) \equiv \sum_{m_{1}} \Sigma_{m_{1} m_{1} ; 12}^{r}(\epsilon) J_{l+m_{1}}\left(\alpha_{R}\right) J_{l^{\prime}-m_{1}}\left(\alpha_{R}\right)
$$

By using the Green's functions $G_{m n ; 11}^{r}, \mathbf{g}_{m n}^{r}$, the self-energy $\Sigma_{m n}^{r}$, and the Dyson's equation, Eq. (25), one obtains the Green's function $G_{m n ; 12}^{r}(\epsilon)$ :

$$
G_{m n ; 12}^{r}=\sum_{l, l^{\prime}} \widetilde{G}_{l}(\epsilon) \frac{J_{l+m}\left(\alpha_{R}\right) J_{l^{\prime}-n}\left(\alpha_{R}\right) \widetilde{\Sigma}_{l l^{\prime}}}{\epsilon+\epsilon_{d \downarrow}+l^{\prime} \omega-\widetilde{\Sigma}_{l^{\prime}}} .
$$

After taking the reverse Fourier transformation, the Green's functions $\mathbf{G}^{r}\left(t, t^{\prime}\right)_{11}$ and $\mathbf{G}^{r}\left(t, t^{\prime}\right)_{12}$ can be obtained immediately. Then substituting them into Eq. (13), $\mathbf{A}_{L(R)}(\epsilon, t)$ and $\mathbf{B}_{L}(\epsilon, t)$ are easily solved

$$
\begin{aligned}
& \mathbf{A}_{L}(\epsilon, t)_{11}= \sum_{n, m, m^{\prime}} G_{m-n, m ; 11}^{r} J_{m}\left(\frac{W_{L}}{\omega}\right) J_{m^{\prime}}\left(\frac{W_{L}}{\omega}\right) \\
& \times e^{-i\left(m-n-m^{\prime}\right) \omega t}, \\
& \mathbf{A}_{R}(\epsilon, t)=\sum_{n} \mathbf{G}_{-n, 0}^{r}(\epsilon) e^{-i n \omega t},
\end{aligned}
$$

$\mathbf{B}_{L}(\epsilon, t)_{12}$

$$
=\sum_{n, m, m^{\prime}} G_{-m-n,-m ; 12}^{r} J_{m}\left(\frac{W_{L}}{\omega}\right) J_{m^{\prime}}\left(\frac{W_{L}}{\omega}\right) e^{i\left(n+m-m^{\prime}\right) \omega t} .
$$

Finally, substituting the expressions of $\mathbf{A}_{L(R)}(\epsilon, t)$ and $\mathbf{B}_{L}(\epsilon, t)$, Eq. (32), into Eq. (14), the time-dependent current $j_{L \uparrow}(t)$ can be obtained. From the definition of the time average,

$$
\langle F(t)\rangle \equiv \lim _{T \rightarrow \infty} \frac{1}{T} \int_{-T / 2}^{T / 2} F(t) d t
$$

the average current $\left\langle j_{L \uparrow}(t)\right\rangle=\left\langle j_{\uparrow}\right\rangle$ can be expressed as

$$
\left\langle j_{\uparrow}\right\rangle=I_{A}+I_{1}+I_{2}+I_{3},
$$

in which

$$
\begin{aligned}
I_{A}= & e \int \frac{d \epsilon}{2 \pi} \Gamma_{L}^{2} \sum_{l, l^{\prime}} \frac{\left|\widetilde{G}_{l}(\epsilon) \widetilde{\Sigma}_{l l^{\prime}}\right|^{2}}{\left|\epsilon+\epsilon_{d \downarrow}+l^{\prime} \omega-\widetilde{\Sigma}_{l^{\prime}}\right|^{2}} \\
& \times\left\{f_{L}(\epsilon) J_{l}^{2}\left(\alpha_{L}\right)-\left[1-f_{L}(-\epsilon)\right] J_{l^{\prime}}^{2}\left(\alpha_{L}\right)\right\},
\end{aligned}
$$

$$
\begin{aligned}
I_{1}=e \int \frac{d \epsilon}{2 \pi} \Gamma_{L} \Gamma_{R} \sum_{l}\left|\widetilde{G}_{l}(\epsilon)\right|^{2}\left\{f_{L}(\epsilon) J_{l}^{2}\left(\alpha_{L}\right)\right. \\
\left.\times \sum_{m} J_{l+m}^{2}\left(\alpha_{R}\right) \widetilde{\rho}_{R}(\epsilon+m \omega)-f_{R}(\epsilon) \widetilde{\rho}_{R}(\epsilon) J_{l}^{2}\left(\alpha_{R}\right)\right\}, \\
I_{2}=e \int \frac{d \epsilon}{2 \pi} \Gamma_{L} \Gamma_{R} \sum_{l, l^{\prime}} \frac{\left|\widetilde{G}_{l}(\epsilon) \widetilde{\Sigma}_{l l^{\prime}}\right|^{2}}{\left|\epsilon+\epsilon_{d \downarrow}+l^{\prime} \omega-\widetilde{\Sigma}_{l^{\prime}}\right|^{2}} \\
\quad \times\left\{f_{L}(\epsilon) J_{l}^{2}\left(\alpha_{L}\right) \sum_{m} J_{l^{\prime}-m}^{2}\left(\alpha_{R}\right) \widetilde{\rho}_{R}(\epsilon+m \omega)\right. \\
\left.\quad-f_{R}(\epsilon) \widetilde{\rho}_{R}(\epsilon) J_{l^{\prime}}^{2}\left(\alpha_{R}\right)\right\},
\end{aligned}
$$$$
\begin{aligned}
I_{3}= & -2 e \int \frac{d \epsilon}{2 \pi} \Gamma_{L} \Gamma_{R} \operatorname{Re} \sum_{l, l^{\prime}} \frac{\left|\widetilde{G}_{l}(\epsilon)\right|^{2} \widetilde{\Sigma}_{l l^{\prime}}^{*}}{\epsilon+\epsilon_{d \downarrow}+l^{\prime} \omega-\widetilde{\Sigma}_{l^{\prime}}^{*}} \\
& \times\left\{f_{L}(\epsilon) J_{l}^{2}\left(\alpha_{L}\right) \sum_{m} J_{l+m}\left(\alpha_{R}\right) J_{l^{\prime}-m}\left(\alpha_{R}\right) \frac{\Delta}{|\epsilon+m \omega|}\right. \\
& \left.\times \widetilde{\rho}_{R}(\epsilon+m \omega)-f_{R}(\epsilon) J_{l}\left(\alpha_{R}\right) J_{l^{\prime}}\left(\alpha_{R}\right) \widetilde{\rho}_{R}(\epsilon) \frac{\Delta}{|\epsilon|}\right\},
\end{aligned}
$$

here $\alpha_{L} \equiv\left(W_{d}-W_{L}\right) / \omega$. The formulas of the average current, Eqs. (33) and (34), are the central results of this paper. It can be applied to the nonlinear response regime, i.e., high bias voltage or high strength of the external fields. In the case of $\Delta=0$, i.e., the right lead is normal, $\widetilde{\Sigma}_{l l^{\prime}}(\epsilon)=0$, then the currents $I_{A}, I_{2}$, and $I_{3}$ are zero, and the current $\left\langle j_{\uparrow}\right\rangle$ reduces to Eq. (6) of Ref. 2. By using the relations $\widetilde{G}_{l}(\epsilon)$ $=\widetilde{G}_{0}(\epsilon-l \omega), \quad \widetilde{\Sigma}_{l}(\epsilon)=\widetilde{\Sigma}_{0}(\epsilon+l \omega)$, and $\widetilde{\Sigma}_{l l^{\prime}}(\epsilon)=\widetilde{\Sigma}_{0, l+l^{\prime}}(\epsilon$ $-l \omega)$, the average current $\left\langle j_{\uparrow}(t)\right\rangle$ can be rewritten as

$$
\begin{aligned}
& I_{A}= e \int \frac{d \epsilon}{2 \pi} \Gamma_{L}^{2}\left|\widetilde{G}_{0}(\epsilon)\right|^{2} \sum_{l, l^{\prime}, m} \frac{\left|\widetilde{\Sigma}_{0 l^{\prime}}(\epsilon)\right|^{2} J_{m}^{2}\left(\alpha_{L}\right) J_{l}^{2}\left(\alpha_{L}\right)}{\left|\epsilon+\epsilon_{d \downarrow}+l^{\prime} \omega-\widetilde{\Sigma}_{l^{\prime}}(\epsilon)\right|^{2}} \\
& \times\left(f_{L}(\epsilon+l \omega)-\left\{1-f_{L}\left[-\epsilon-\left(l^{\prime}-m\right) \omega\right]\right\}\right), \\
& I_{1}=e \int \frac{d \epsilon}{2 \pi} \Gamma_{L} \Gamma_{R}\left|\widetilde{G}_{0}(\epsilon)\right|^{2} \sum_{l, m} J_{m}^{2}\left(\alpha_{R}\right) J_{l}^{2}\left(\alpha_{L}\right) \widetilde{\rho}_{R}(\epsilon+m \omega) \\
& \times\left\{f_{L}(\epsilon+l \omega)-f_{R}(\epsilon+m \omega)\right\}, \\
& I_{2}=e \int \frac{d \epsilon}{2 \pi} \Gamma_{L} \Gamma_{R}\left|\widetilde{G}_{0}(\epsilon)\right|^{2} \sum_{l, l^{\prime}, m} \\
& \quad \times \frac{\left|\widetilde{\Sigma}_{0 l^{\prime}}(\epsilon)\right|^{2} J_{l}^{2}\left(\alpha_{L}\right) J_{l^{\prime}-m}^{2}\left(\alpha_{R}\right)}{\left|\epsilon+\epsilon_{d \downarrow}+l^{\prime} \omega-\widetilde{\Sigma}_{l^{\prime}}(\epsilon)\right|^{2}} \\
& \times \widetilde{\rho}_{R}(\epsilon+m \omega)\left\{f_{L}(\epsilon+l \omega)-f_{R}(\epsilon+m \omega)\right\},
\end{aligned}
$$




$$
\begin{aligned}
I_{3}= & -2 e \operatorname{Re} \int \frac{d \epsilon}{2 \pi} \Gamma_{L} \Gamma_{R}\left|\widetilde{G}_{0}(\epsilon)\right|^{2} \sum_{l, l^{\prime}, m} \\
& \times \frac{\widetilde{\Sigma}_{0 l^{\prime}}^{*}(\epsilon) J_{l}^{2}\left(\alpha_{L}\right) J_{l^{\prime}-m}\left(\alpha_{R}\right) J_{m}\left(\alpha_{R}\right)}{\epsilon+\epsilon_{d \downarrow}+l^{\prime} \omega-\widetilde{\Sigma}_{l^{\prime}}^{*}(\epsilon)} \frac{\Delta}{|\epsilon+m \omega|} \\
& \times \widetilde{\rho}_{R}(\epsilon+m \omega)\left\{f_{L}(\epsilon+l \omega)-f_{R}(\epsilon+m \omega)\right\} .
\end{aligned}
$$

From Eq. (35), various processes of the PAT and the PAAT can be seen clearly. The current $I_{A}$ includes all kinds of the PAAT processes; for example, an electron from the left lead tunnels into the dot by $l$ photon emission, followed by an Andreev reflection via $l^{\prime}$ photon absorption, and finally an Andreev-reflected hole absorbs $m$ photons to tunnel back to the left lead, etc. The currents $I_{1}, I_{2}$, and $I_{3}$ include all PAT processes. It should be pointed out that in the case of zero temperature and $|e V|<\Delta\left(e V=\mu_{L}-\mu_{R}\right.$ is the dc bias), the currents $I_{1}, I_{2}$, and $I_{3}$ are zero in the absence of the external fields, but they are nonzero in the presence of the external fields due to the PAT processes.

In the following three sections we shall apply our current formulas, Eqs. (33) and (35), to investigate the dependence of the average current $\langle j\rangle=\left\langle j_{\uparrow}\right\rangle+\left\langle j_{\downarrow}\right\rangle$ on the gate voltage $v_{g}$ and the dc bias $V$.

\section{THE CASE OF $\alpha_{L}=0$}

In this section we investigate the case of $\alpha_{L}=0$, i.e., external fields are applied to both the left lead and the dot with equal amplitudes $\left(W_{L}=W_{d}\right)$. It is equivalent to the case in which the external field is applied only on the right superconducting lead, ${ }^{2}$ and the photon-assisted processes occur only around the right barrier region. In the numerical calculations, we make further simplifications: (1) Assume that $\epsilon_{d \sigma}(t)$ be independent of $\sigma$ (i.e., $\epsilon_{d \uparrow}=\epsilon_{d \downarrow} \equiv \epsilon_{d}$ ), then $\left\langle j_{\uparrow}\right\rangle$ $=\left\langle j_{\downarrow}\right\rangle=\frac{1}{2}\langle j\rangle$. (2) Set the temperature $\mathcal{T}$ to be zero. (3) Consider two symmetric barriers, i.e., $\Gamma_{L}=\Gamma_{R}$. (4) Let the gate voltage $v_{g}$ control the intradot level as $\boldsymbol{\epsilon}_{d}\left(v_{g}\right)=\boldsymbol{\epsilon}_{d}(0)$ $-e v_{g}$. In calculation, we take $\Delta=1$ and the units of $e=1$. In the following, we shall discuss two cases of $\hbar \omega<\Delta$ and $\hbar \omega>\Delta$ in detail.

\section{A. $\hbar \omega<\Delta$}

We first investigate the case with zero bias $(V=0)$, and choose the parameters $\omega=0.2$ and set $\epsilon_{d}=0$ when $v_{g}=0$. Figure 1 shows the average current $\langle j\rangle$ vs the gate voltage $v_{g}$, which controls the electronic level $\epsilon_{d}$ of the dot. We have assumed that the frequency $(\omega=0.2)$ is much smaller than the gap $(\Delta=1)$ in this subsection. Then the currents $I_{1}$, $I_{2}$, and $I_{3}$ are small, and the Andreev current $I_{A}$ dominates, leading to the following features: (1) A series of negative peaks emerge on the left-hand side of the original resonant peak, which appears originally at the small bias but without external fields, while a series of positive peaks on its righthand side. Notice that the negative peaks are originated from the electron-photon pump effect, ${ }^{39}$ i.e., in the presence of the external field, electrons may transit from the region of higher voltage to the region of lower voltage by absorbing photons, which leads to a negative current. (2) The spacing of the

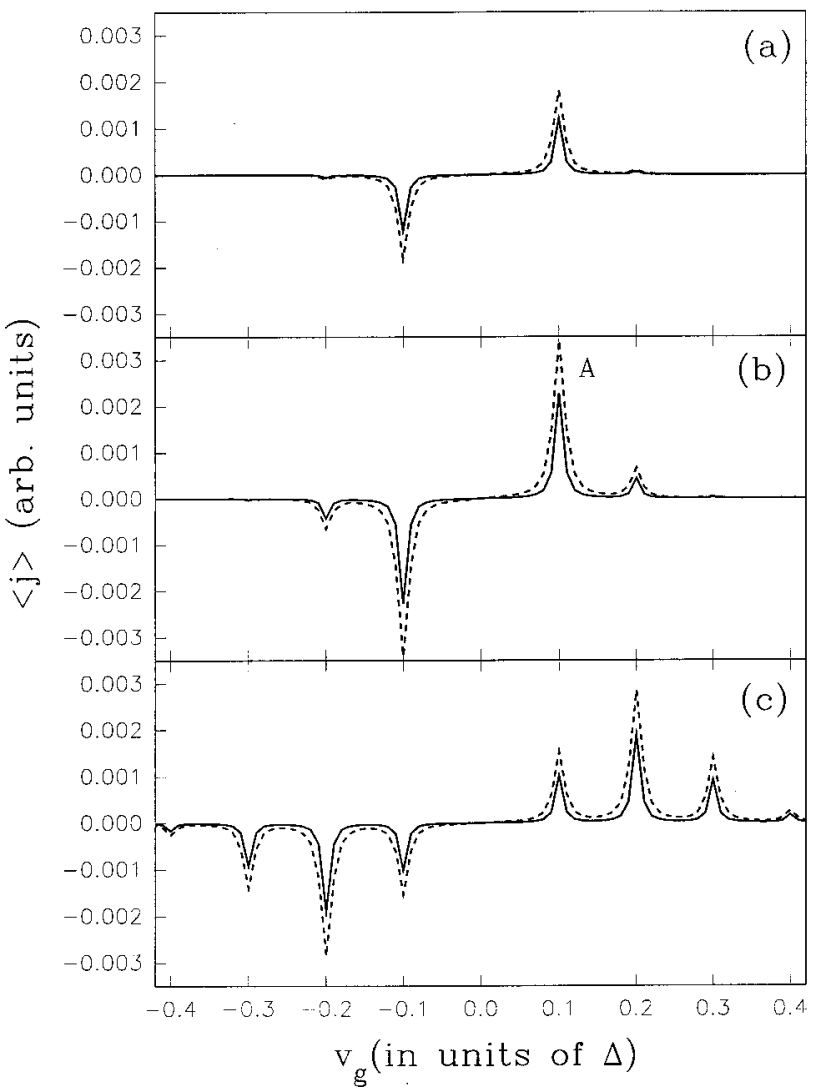

FIG. 1. The average current $\langle j\rangle$ vs the gate voltage $v_{g}$ at the zero bias; the other parameters are $\omega=0.2, \Delta=1, \alpha_{L}=0, \Gamma_{L}=\Gamma_{R}$ $=0.01$ (solid curves), $\Gamma_{L}=\Gamma_{R}=0.015$ (dotted curves), and $\epsilon_{d}=0$ when $v_{g}=0$. (a), (b), and (c) correspond to different amplitudes of external fields: $\alpha_{R}=0.4,0.7$, and 1.5 , respectively.

neighboring peaks is $\frac{1}{2} \hbar \omega$. (3) With increasing the amplitude of external fields, more and more peaks emerge, but the heights of the peaks increase nonmonotonically. (4) At zero bias, $V=0$, the original resonant peak disappears. Features (1) and (2) appear only in $N$-QD- $S$ system but not in $N$-QD- $N$ system. ${ }^{2,4,9}$ In fact, they are originated from the PAAT. If the gate voltage $v_{g}=-0.1$, the intradot energy level $\epsilon_{d}$ will be at 0.1 above the left and the right chemical potential $\left(\mu_{L}, \mu_{R}\right)$ [see Fig. 2(a)], then a hole in the left lead with the energy $\epsilon$ about 0.1 can tunnel through the left bar-
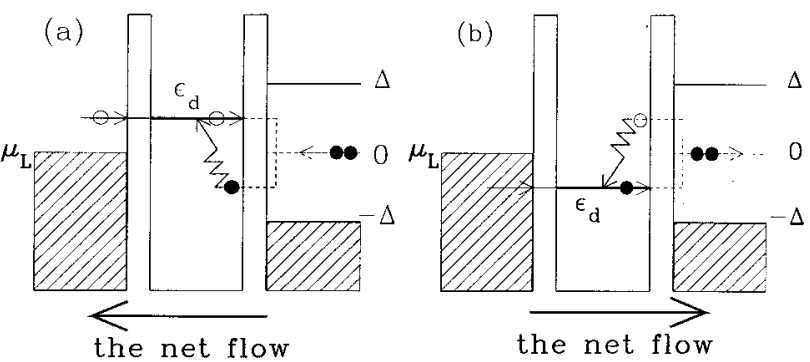

FIG. 2. A schematic diagram for the photon-assisted Andreev tunneling (PAAT): (a) An incident hole leads to an Andreevreflected electron, leading to a negative current. (b) an incident electron leads to an Andreev-reflected hole, leading to a positive current. 


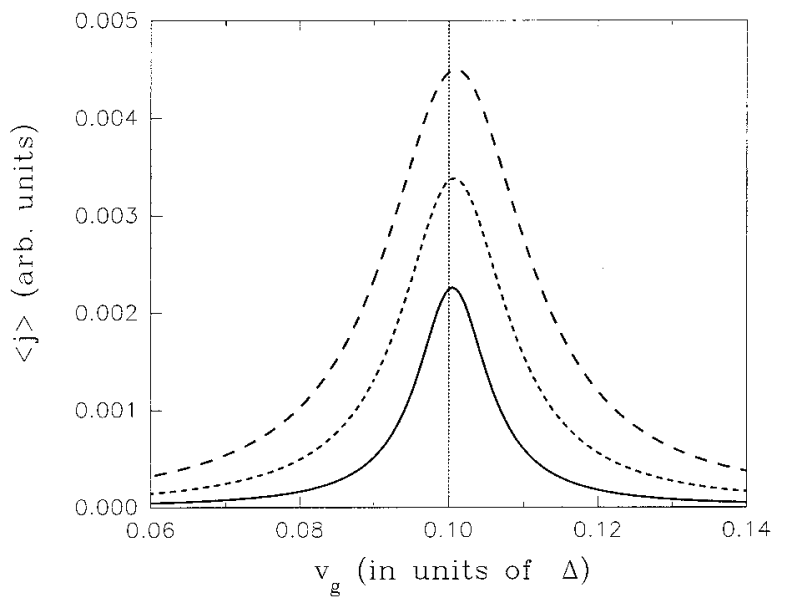

FIG. 3. The detailed line shape of the " $A$ " peak in Fig. 1(b). The solid, dotted, and dashed curves correspond to $\Gamma_{L}=\Gamma_{R}$ $=0.01,0.015$, and 0.02, respectively. The other parameters are the same as in Fig. 1(b).

rier into the dot, and reach the right barrier. In the absence of external fields, this hole cannot experience an Andreev reflection since no state is available at $\epsilon=-0.1$. However, in the presence of external fields, an Andreev reflected electron with the energy $\epsilon=-0.1$ can absorb a photon and jump to the state $\epsilon_{d}$ [see Fig. 2(a)]. So the Andreev reflection can occur with the help of a photon absorption; meanwhile a Cooper pair in the right lead will be destroyed, leading to a negative current. If the change of $v_{g}$ exceeds a certain value (about several $\Gamma$ ), the level $\epsilon_{d}$ will move out of the range in which the condition of the Andreev reflection is satisfied, then the PAAT processes mentioned above are forbidden, and the current $I_{A}$ drops to almost zero. This explains why a negative peak emerges in the curve of $\langle j\rangle$ vs $v_{g}$ at $v_{g}=$ -0.1 . Similarly, when $v_{g}=0.1$, the level $\epsilon_{d}$ will move down to -0.1 below both $\mu_{L}$ and $\mu_{R}$, now an electron from the left lead can tunnel into the dot, followed by an Andreev reflection via a photon emission [see Fig. 2(b)], leading to a positive peak in the curve of $\langle j\rangle$ vs $v_{g}$. With the increase of external fields, the multiple photon-assisted Andreev tunneling may also happen. As for the interval between neighboring peaks, $\frac{1}{2} \hbar \omega$, it is easy to understand just by noticing that each change of $\frac{1}{2} \hbar \omega$ for the gate voltage $v_{g}$ corresponds to a variation from the process with $n$-PAAT to $(n+1)$-PAAT.

In order to investigate the detailed line shape of the PAAT peak, in Fig. 3 we present the amplified peak corresponding to the peak marked with " $A$ " in Fig. 1(b). One can clearly see that the top of the peak is flat (i.e., zero derivative), not as sharp (i.e., undefined derivative); and the half width of the peak is determined by the linewidth $\Gamma_{L}$. Notice that the positions of the PAAT peaks are not exactly at $(n / 2) \hbar \omega(n=$ $\pm 1, \pm 2, \ldots$ ), but slightly shifted from them: towards the right direction for positive peaks (see Fig. 3), while the left direction for negative peaks (not shown here). The reason of this slight deviation comes from that the real part of $\Sigma_{R, m n}^{r}(\epsilon)$ is not exactly zero.

Now, let us consider the nonzero bias case. Then the original resonant peak will emerge even at very small bias (about several Г) [see Fig. 4(a)], which is originated from the Andreev tunneling without the help of photons. With the

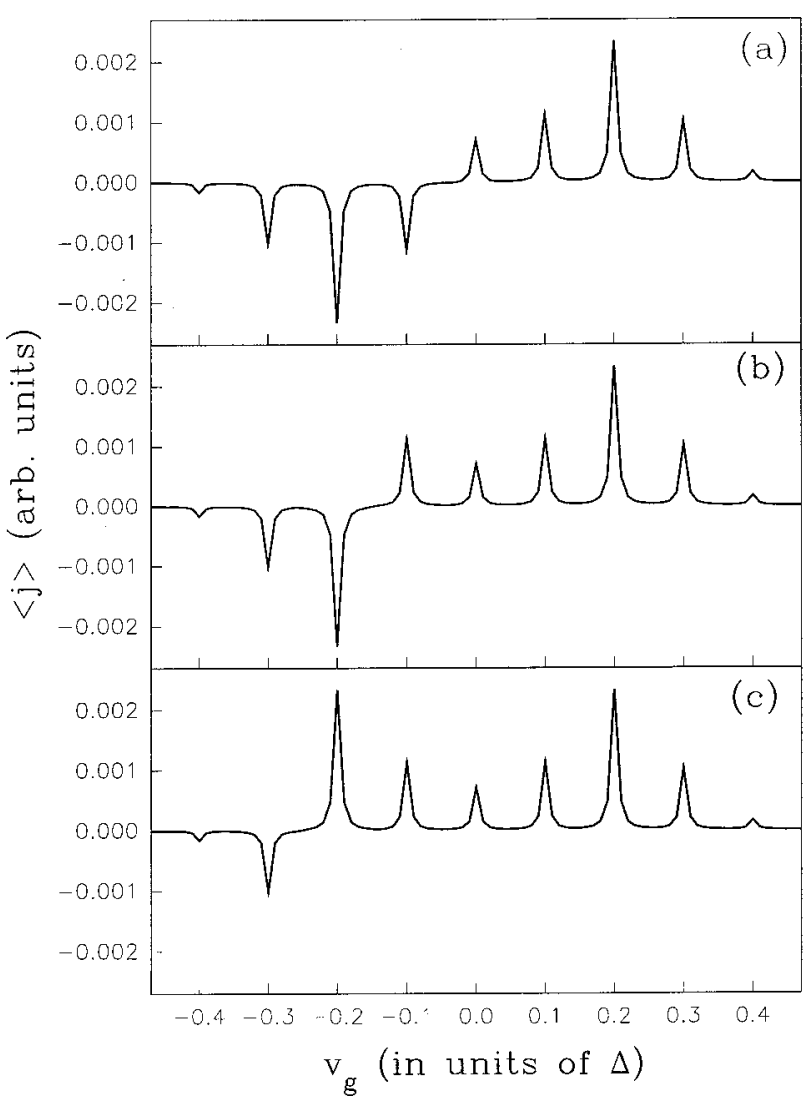

FIG. 4. The average current $\langle j\rangle$ vs the gate voltage $v_{g}$ at $\alpha_{L}$ $=0$ and $\alpha_{R}=1.5$. (a), (b), and (c) correspond to the different bias: $V=0.02,0.15$, and 0.25 , respectively. The other parameters are $\omega$ $=0.2, \Delta=1, \Gamma_{L}=\Gamma_{R}=0.01$, and $\epsilon_{d}=0$ when $v_{g}=0$.

increase of the bias $V$, the curve of $\langle j\rangle$ vs $v_{g}$ has almost no change at the beginning, until the bias $V$ reaches $\frac{1}{2} \hbar \omega$ $=0.1$. When $V$ changes from $V<0.1$ to $V>0.1$, the peak at $v_{g}=-0.1$ changes from negative to positive [see Fig. 4(b)], due to the fact that for $V<0.1(V>0.1)$, the state with $\epsilon$ $=0.1$ in the left lead is occupied by a hole (an electron), resulting in a hole-type (an electron-type) PAAT. Similarly, when $V$ increases from $V<0.2$ to $V>0.2(\hbar \omega=0.2)$, the two-photon PAAT peak will change from negative to positive too [see Fig. 4(c)].

\section{B. $\hbar \omega>\Delta$}

In this subsection, we investigate the case of $\hbar \omega>\Delta$. As mentioned in Sec. II, although the upper limit of the frequency of the external field is restricted by the adiabatic approximation, it still can reach up to tens of $\mathrm{THz}$ or even higher according to the estimation. ${ }^{1}$ If one takes $1 \mathrm{mev}$ as the energy gap $\Delta$ of the superconductor, then it corresponds to a photon frequency of $250 \mathrm{GHz}$, which is still much smaller than the upper limit of the frequency of the adiabatic approximation. Therefore, the condition of $\hbar \omega>\Delta$ is reasonable.

In the case of $\hbar \omega>\Delta$, even if $|e V|<\Delta$, the part of $I_{1}$ $+I_{2}+I_{3}$ has significant contribution to the average current $\langle j\rangle$ due to the PAT processes. Figure 5(a) shows the average current $\langle j\rangle$ vs the gate voltage $v_{g}$ for $\hbar \omega=1.5$ and $V=0.2$. In addition to the Andreev-tunneling peak (including the 


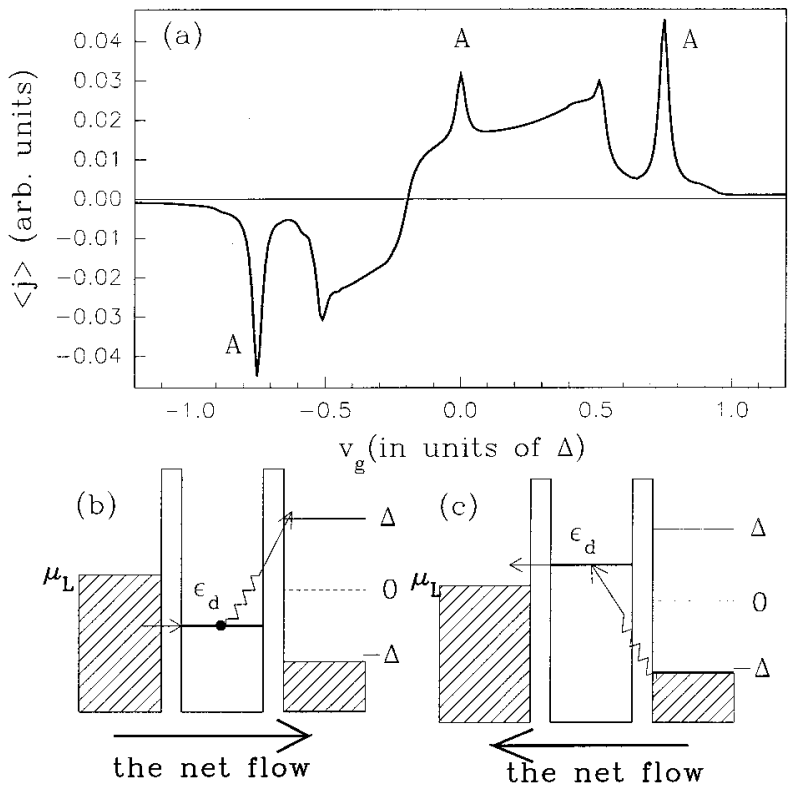

FIG. 5. (a) The average current $\langle j\rangle$ vs the gate voltage $v_{g}$ for the frequency $\omega=1.5$, where $\alpha_{L}=0, \alpha_{R}=1.1, V=0.2, \Delta=1, \Gamma_{L}=\Gamma_{R}$ $=0.04$, and $\epsilon_{d}=0$ when $v_{g}=0$. (b) and (c) are the schematic diagrams for the PAT processes. The net electron flow is from left to right in (b), while from right to left in (c).

photon-assisted and nonphoton-assisted, marked by " $A$ "), the extra positive current emerges for $-0.2<v_{g}<0.5$, and the extra negative current emerges when $-0.5<v_{g}<-0.2$. The extra current comes from the normal photon-assisted tunneling, which can be explained as follows: (1) When the gate voltage $v_{g}=0.5$, the intradot energy level $\epsilon_{d}$ will be at -0.5 . Then the electron at the level $\epsilon_{d}$ can just jump to a quasiparticle state above the gap in the right lead via a photon absorption [see Fig. 5(b)], leading to a large positive current. (2) With the decrease of the gate voltage $v_{g}$, the level $\epsilon_{d}$ moves up, then an electron must transit to higher quasiparticle's state where the density of states of the superconducting electrode $\rho_{R}^{S}(\epsilon)$ is smaller than the value near the edge of the gap. So the current $\langle j\rangle$ slowly descends [see Fig. 5(a)]. (3) When $v_{g}$ changes from $v_{g}>-0.2$ to $v_{g}<-0.2$, the level $\epsilon_{d}$ will change from an occupied state to an unoccupied state, and the current $\langle j\rangle$ varies from positive to negative [see Fig. 5(a)]. (4) For $-0.5<v_{g}<-0.2$, the current $\langle j\rangle$ is negative, because the intradot level $\epsilon_{d}$ now is empty, and a quasiparticle below $-\Delta$ in the right lead can first absorb a photon to transit into the intradot level $\epsilon_{d}$ [see Fig. 5(c)], then tunnel to the left lead, leading to a negative current.

Next we investigate the case with much higher frequency of external fields $(\omega=3>2 \Delta)$. The average current $\langle j\rangle$ vs the gate voltage $v_{g}$ is shown in Fig. 6(a). The following features can be seen clearly: (1) The PAAT peak at $v_{g}=$ $\pm \frac{1}{2} \hbar \omega$ are very small, since the probability of the Andreev reflection is much smaller for the energy $\epsilon_{d}>\Delta .{ }^{39}$ (2) At $v_{g}=\hbar \omega-\Delta$, the current $\langle j\rangle$ has a rapid increase within the range of several $\Gamma$, then it slowly descends with the decrease of $v_{g}$, due to the PAT processes as shown in Fig. 5(b). (3) Around $v_{g}=\Delta$, the current $\langle j\rangle$ abruptly increases about twice as much with the decrease of $v_{g}$. This is because when $v_{g}$ $>\Delta$, the level $\epsilon_{d}$ is moved down below $\mu_{L}$ and $-\Delta$, an

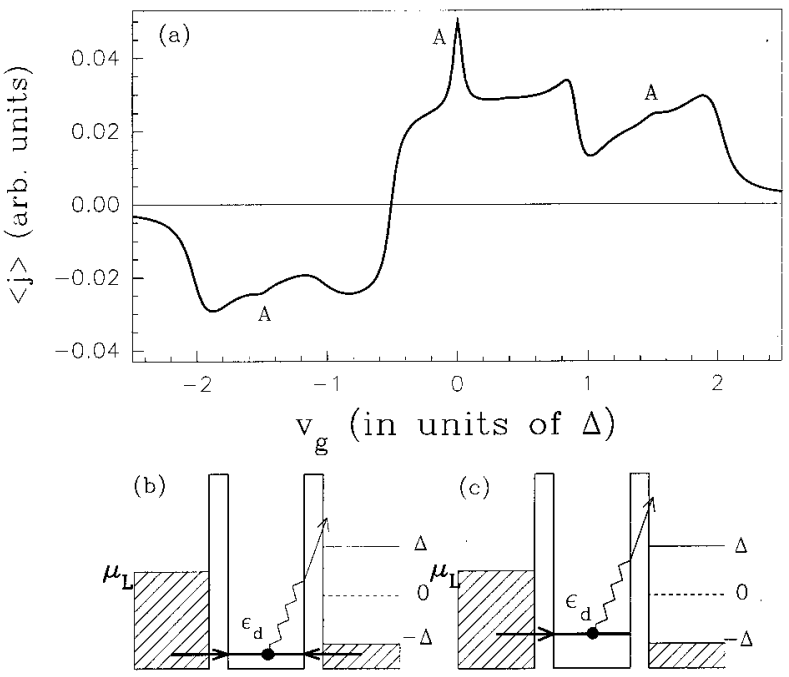

FIG. 6. (a) The average current $\langle j\rangle$ vs the gate voltage $v_{g}$ for the frequency $\omega=3$, where $\alpha_{L}=0, \alpha_{R}=1.1, V=0.5, \Delta=1, \Gamma_{L}=\Gamma_{R}$ $=0.08$, and $\epsilon_{d}=0$ when $v_{g}=0$. (b) and (c) are the schematic diagrams for abruptly increasing of the current while $v_{g}$ varies from $v_{g}>\Delta$ to $v_{g}<\Delta$. Only the electron in the left lead can tunnel to the dot in (c), instead, in (b), the electrons both in the left lead and in the right lead can tunnel into the dot.

electron can only tunnel out of the dot through the right barrier to the right lead via photon absorption (notice that the photon absorption or emission cannot occur in the left barrier due to $\alpha_{L}=0$ ), then the electrons in the left and the right lead with energy $\epsilon=\epsilon_{d}$ can tunnel into the dot [see Fig. 6(b)]. However, when $v_{g}<\Delta$, the level $\epsilon_{d}$ moves up above $-\Delta$, and only the electron in the left lead can tunnel into the dot [see Fig. 6(c)], so the net electron flow of $v_{g}<\Delta$ is about twice the value for $v_{g}>\Delta$.

\section{THE EXTERNAL FIELD APPLIED ONLY TO THE DOT}

In this case, $\alpha_{L}=\alpha_{R}$, i.e., an electron tunneling through either the left or the right barrier can absorb or emit photons; therefore the process becomes more complicated than the case discussed in Sec. III. For the $N$-QD- $N$ system, which has been investigated extensively, ${ }^{1-5,9,10,15}$ basic features of the current vs the gate voltage at the small bias are a series of side-band peaks with equal interval $\hbar \omega$ emerges, the height of the peak is proportional to the square of the Bessel's function $J_{n}^{2}(\alpha)$, where $\alpha=W_{d} / \omega$. The negative current (i.e., the electron-photon pumping effect) does not emerge if the external field only applied to the dot. In contrast, the $N$-QD- $S$ system is very different. In the following, we discuss two cases with $\hbar \omega<\Delta$ and $\hbar \omega>\Delta$.

Figure 7 shows the average current $\langle j\rangle$ vs the gate voltage $v_{g}$ for $\hbar \omega<\Delta$. A series of peaks emerges with positive value for $v_{g}>0$, and negative for $v_{g}<0$, respectively. Notice that the interval is $\frac{1}{2} \hbar \omega$, not $\hbar \omega$. For the small bias, an extra peak emerges at $v_{g}=0$. These peaks are originated from the PAAT as shown in Fig. 2. In comparison with the case of $\alpha_{L}=0$ (the dotted line in Fig. 7), the heights of the onephoton- and the two-photon-PAAT peak are much lower, but 


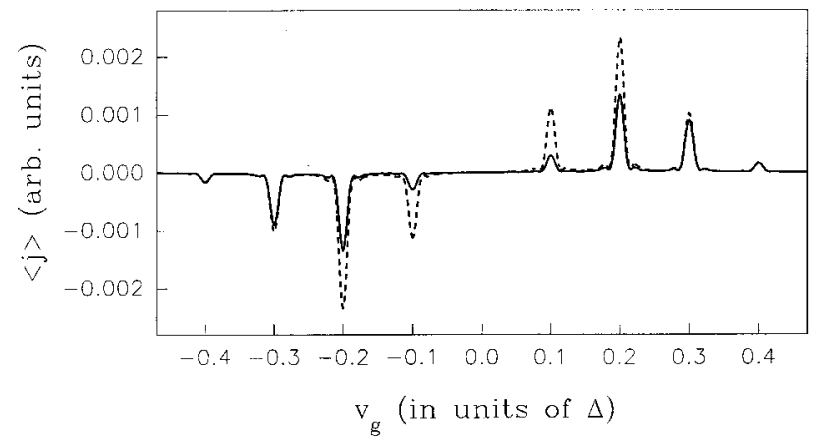

FIG. 7. The solid curve describes the current $\langle j\rangle$ vs the gate voltage $v_{g}$ in the case of $\alpha_{L}=\alpha_{R}=1.5$. The dotted curve shows $\langle j\rangle$ vs $v_{g}$ at $\alpha_{L}=0$ for comparison. The other parameters are the same as the solid curve in Fig. 1(c).

for the higher-order PAAT peaks with more photons involved, the heights almost do not change, which is easily understood from the PAT that occurred in the left barrier.

Now let us consider the case of $\hbar \omega>\Delta$, in which the current $\langle j\rangle$ vs the gate voltage $v_{g}$ becomes quite complicated [see Fig. 8(a)]. Various PAT and PAAT processes will occur, and the average current $\langle j\rangle$ changes alternatively between positive and negative values. Peaks marked by " $A$ " are the $n$-photon $(n=0, \pm 1)$ PAAT peaks. In the following we discuss them in detail but only consider the single photon processes: (1) When $v_{g}<-1.7$, i.e., $v_{g}<-V-\hbar \omega$, the energy level $\epsilon_{d}$ is far above both $\mu_{L}$ and $\Delta$, no PAT process can occur, and the current $\langle j\rangle$ is almost zero. (2) At $v_{g}=-1.7$, an incident electron at the Fermi surface of the left lead can tunnel into the intradot level $\epsilon_{d}$ via a photon absorption, then tunnel out of the dot through the left (the right) barrier to the left (the right) lead [see Fig. 8(b)], resulting in an electron

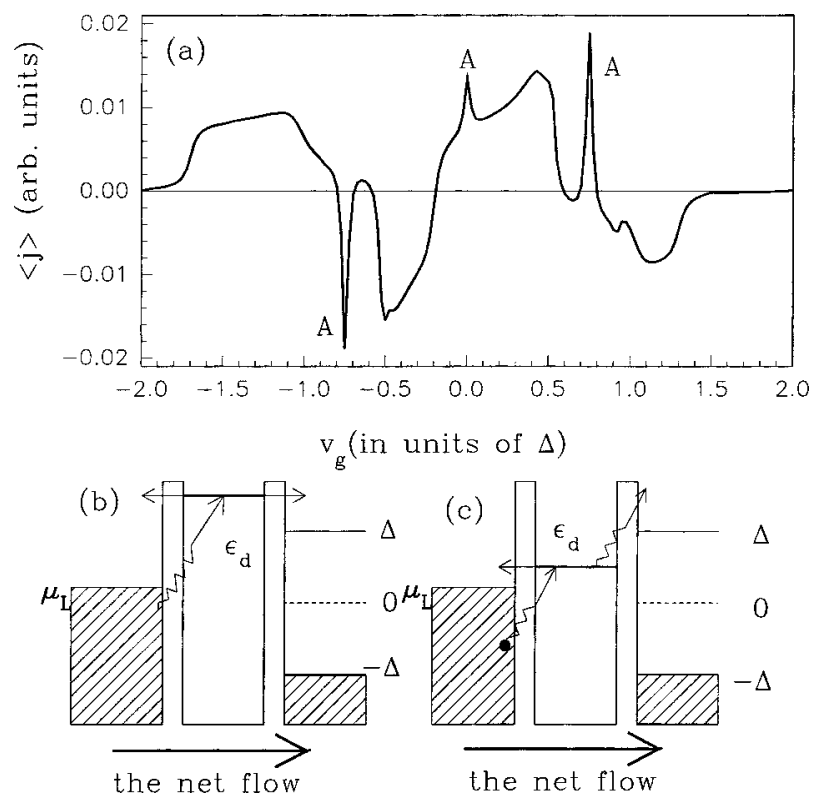

FIG. 8. (a). The average current $\langle j\rangle$ vs the gate voltage $v_{g}$ in $\alpha_{L}=\alpha_{R}=1.1$, the other parameters are the same as in Fig. 5(a). (b) and (c) are the schematic diagrams for the PAT processes, corresponding to the cases of $-1.7<v_{g}<-1.0$ and $-1.0<v_{g}<-0.5$, respectively.

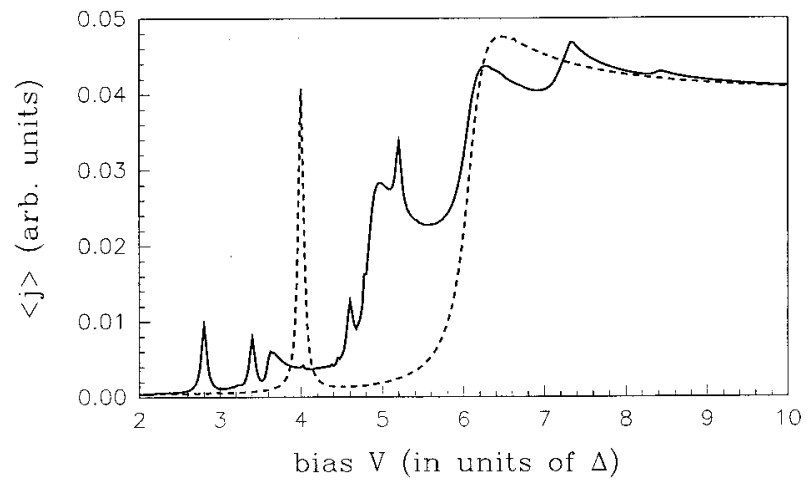

FIG. 9. The average current $\langle j\rangle$ vs the bias $V$, where $\alpha_{L}=0$, $\alpha_{R}=1.1, \omega=0.6, \Delta=1, \Gamma_{L}=\Gamma_{R}=0.04$, and $\epsilon_{d}=-2$ when $V=0$. The dotted curve corresponds to the case without external fields (i.e., $\alpha_{L}=\alpha_{R}=0$ ) for comparison.

flow from the left lead to the right lead. So the current increases abruptly within the energy range of several $\Gamma$. (3) While $v_{g}$ varies from -1.7 to $-1.0(=-\Delta)$, the level $\epsilon_{d}$ is gradually pulled down, and the corresponding density of states of the right superconducting lead increases, leading to a larger tunneling probability of the electron from the dot to the right lead. So, the current $\langle j\rangle$ is increased slowly. (4) When $v_{g}$ is larger than -1.0 , the level $\epsilon_{d}$ will be below the gap $\Delta$; then the electron tunneling from the dot to the right lead is prohibited, leading to an abrupt drop of the current. (5) For $-1.0<v_{g}<-0.5$, the electron in the left lead can jump to the level $\epsilon_{d}$ in the dot via a photon absorption, then absorbing another photon to transit to the right lead [see Fig. $8(\mathrm{c})$ ], so the current has a small positive value when $v_{g}$ is out of the range of the Andreev-reflection peak "A." (6) While $v_{g}$ crosses -0.5 (i.e., $\left.\Delta-\hbar \omega\right)$, the PAT processes as shown in Fig. 5(c) occur and lead to a very big negative current. (7) While $v_{g}$ varies from -0.5 to -0.2 (i.e., $-V$ ), the magnitude of the negative current gradually decreases due to the decrease of the density of states $\rho_{R}^{S}(\epsilon)$. (8) When $v_{g}$ across -0.2 , the level $\epsilon_{d}$ passes across the Fermi surface of the left lead, and will always be occupied by electrons. Then PAT process mentioned in (6) is prohibited. Instead, another kind of PAT process as shown in Fig. 5(b) occurs, leading to a strong variation of the current from negative to positive. For $v_{g}>0$, one can understand the behavior of the current similarly.

\section{THE I-V CHARACTERISTICS}

In this section, we investigate the dependence of the current $I$ on the bias $V$. For symmetric barriers, we assume that the intradot level $\epsilon_{d}$ varies as $\epsilon_{d}+V / 2$. In the absence of external fields, an extra Andreev-tunneling peak superimposes on a conventional current plateau in the $I-V$ characteristics (see the dotted curve in Fig. 9). However, in the presence of external fields, each Andreev tunneling peak will be split into a series of side-band peaks due to the PAAT, and each current plateau will also be split into a series of substep plateaus due to the PAT. It should be pointed out that the interval of the side-band peaks, $\frac{1}{2} \hbar \omega$, is different from the interval of the substep plateaus, $\hbar \omega$.

Finally, we should stress that the $N$-QD- $S$ system in the 
presence of external fields can be realized experimentally by using the existing technique. In fact, in many experiments the MW fields have been applied on the $N$-QD- $N$ system, either symmetrically (i.e., $\alpha_{L}=\alpha_{R}$, as discussed in Sec. IV) or asymmetrically (as discussed in Sec. III) ${ }^{3-7}$ Many mesoscopic "hybrid" system, including $S$-SQD- $S, N$-SQD- $N$, etc., have been investigated extensively. ${ }^{27,28}$ In particular, Hergenrother et al. have succeeded in applying an external field to a $N$-superconducting island $-N$ system. ${ }^{29}$ It should be able to realize to apply external fields on a $N$-QD- $S$ system. Since we have already proved that the case with the external fields applied on all three regions is completely equivalent to the case with the external fields only applied on the left lead and the dot (see Appendix A), so to apply external fields on the left lead and the dot is enough to have all the physics.

\section{CONCLUSIONS}

In this paper, we have investigated the physics of the electron tunneling through a mesoscopic hybrid $N$-QD- $S$ system. By using the nonequilibrium-Green-function method, the time-dependent current $j_{L}(t)$ and the average current $\langle j\rangle$ are derived. The PAAT processes and the normal PAT processes are investigated in detail. We find in the presence of external fields, the original resonant peak of the average current $\langle j\rangle$ vs the gate voltage $v_{g}$ will be split into a series of the side-band peaks with the equal interval $\frac{1}{2} \hbar \omega$, on the left-hand side of the original resonant peak are the negative peaks, while on the right-hand side are the positive peaks. This is very different from the $N$-QD- $N$ system in the presence of the time-dependent external fields. Due to the existence of the energy gap $\Delta$, the physics of PAT becomes much richer, depending on the frequency $\omega$, the gap $\Delta$, and the bias $V$. As a result, the current $\langle j\rangle$ vs $v_{g}$ becomes more complicated, and can vary many times between the positive and the negative. Moreover, in the $I-V$ characteristics, the Andreev-reflection peak and the current plateau can be split into a series of side-band peaks and substep plateaus.

\section{ACKNOWLEDGMENTS}

We gratefully acknowledge financial support by a RGC grant from the SAR Government of Hong Kong under Grant No. HKU 7112/97P, a grant from the Croucher Foundation, a CRCG grant from the University of Hong Kong, and a research grant from the Chinese National Natural Science Foundation, and the Doctoral Program Foundation of Institution of Higher Education. We also thank the Computer Center of the University of Hong Kong for computational facilities. Q.F.S. and T.S.L. would like to thank B. G. Wang, Q. R. Zheng, X. A. Zhao, and Y. D. Wei for many helpful discussions.

\section{APPENDIX A}

In this appendix, we shall prove that the case with external fields applied to all three regions (the left lead, the right lead, and the dot) is exactly equivalent to the case with the external fields only applied on the left lead and the dot but not on the right superconducting lead. While the right lead is also applied by an external field, under the adiabatic approximation the Hamiltonian $H_{R}$ can be expressed as

$$
\begin{aligned}
H_{R}= & \sum_{p, \sigma}\left[\epsilon_{p}+W_{R}(t)\right] b_{p \sigma}^{\dagger} b_{p \sigma}+\sum_{p}\left[\widetilde{\Delta}^{*} b_{p \downarrow} b_{-p \uparrow}\right. \\
& \left.+\widetilde{\Delta} b_{-p \uparrow}^{\dagger} b_{p \downarrow}^{\dagger}\right] .
\end{aligned}
$$

Notice that in the presence of the external field, not only the energy of the quasiparticle, but the energy of the Cooper pair is affected, so $\widetilde{\Delta}$ in Eq. (A1) becomes

$$
\widetilde{\Delta}=\Delta \exp \left[-\frac{2 i}{\hbar} \int_{0}^{t} W_{R}\left(t^{\prime}\right) d t^{\prime}\right],
$$

where $\Delta$ is the complex order parameter without the external field. Then we perform a unitary transformation with the unitary operator $U$ as

$$
\begin{aligned}
U(t)= & \exp \left\{\frac { i } { \hbar } \int _ { 0 } ^ { t } d t ^ { \prime } W _ { R } ( t ^ { \prime } ) \left[\sum_{k, \sigma} a_{k \sigma}^{\dagger} a_{k \sigma}+\sum_{p, \sigma} b_{p \sigma}^{\dagger} b_{p \sigma}\right.\right. \\
& \left.\left.+\sum_{\sigma} c_{\sigma}^{\dagger} c_{\sigma}\right]\right\} .
\end{aligned}
$$

Under this unitary transformation, an operator $X$ ( $X$ represents $a_{k \sigma}, b_{p \sigma}$, or $c_{\sigma}$ ) changes into

$$
\begin{aligned}
& U(t) X U^{\dagger}(t)=X \exp \left[-\frac{i}{\hbar} \int_{0}^{t} W_{R}\left(t^{\prime}\right) d t^{\prime}\right], \\
& U(t) X^{\dagger} U^{\dagger}(t)=X^{\dagger} \exp \left[\frac{i}{\hbar} \int_{0}^{t} W_{R}\left(t^{\prime}\right) d t^{\prime}\right],
\end{aligned}
$$

and the Hamiltonian $H$ becomes

$$
\begin{aligned}
& H_{L}(t)=\sum_{k, \sigma}\left[\epsilon_{k}+W_{L}(t)-W_{R}(t)\right] a_{k \sigma}^{\dagger} a_{k \sigma}, \\
& H_{\mathrm{dot}}(t)=\sum_{\sigma}\left[\epsilon_{d \sigma}+W_{d}(t)-W_{R}(t)\right] c_{\sigma}^{\dagger} c_{\sigma},
\end{aligned}
$$

$$
\begin{gathered}
H_{R}=\sum_{p, \sigma} \epsilon_{p} b_{p \sigma}^{\dagger} b_{p \sigma}+\sum_{p}\left[\Delta^{*} b_{p \downarrow} b_{-p \uparrow}+\Delta b_{-p \uparrow}^{\dagger} b_{p \downarrow}^{\dagger}\right], \\
H_{T}=\sum_{k, \sigma}\left[L_{k} a_{k \sigma}^{\dagger} c_{\sigma}+L_{k}^{*} c_{\sigma}^{\dagger} a_{k \sigma}\right]+\sum_{p, \sigma}\left[R_{p} b_{p \sigma}^{\dagger} c_{\sigma}+R_{p}^{*} c_{\sigma}^{\dagger} b_{p \sigma}\right] .
\end{gathered}
$$

Obviously, the case with external fields applied to all three regions is exactly equivalent to the case with the external fields only applied to the left lead and the quantum dot with the effective amplitudes $\widetilde{W}_{L}(t)=W_{L}(t)-W_{R}(t)$ and $\widetilde{W}_{d}(t)$ $=W_{d}(t)-W_{R}(t)$. It is clear that if the amplitudes of the external fields are the same in all three regions, no physical effects will exist by external fields. This result is similar to the one of the normal system discussed in Ref. 1.

\section{APPENDIX B}

In this appendix, we present the derivation of the selfenergy $\Sigma_{R}^{r}\left(t_{1}, t_{2}\right)$ and $\Sigma_{R}^{<}\left(t_{1}, t_{2}\right)$. First, we solve the Green's function $\Sigma_{p} g_{p}^{r}\left(t_{1}, t_{2}\right)$. From the Hamiltonian $H_{R}$, we have 


$$
\begin{gathered}
i \frac{\partial}{\partial t} b_{p \uparrow}(t)=\epsilon_{p} b_{p \uparrow}(t)+\Delta b_{-p \downarrow}^{\dagger}(t), \\
i \frac{\partial}{\partial t} b_{-p \downarrow}^{\dagger}(t)=-\epsilon_{p} b_{-p \downarrow}^{\dagger}(t)+\Delta^{*} b_{p \uparrow}(t) .
\end{gathered}
$$

For the system under consideration, only the right lead is a superconductor, the current will not depend on the phase of the order parameter of the superconducting lead, only depend on its modulus, $|\Delta|$. So we assume that $\Delta$ is real. Then the differential equations can be easily solved

$$
\begin{aligned}
b_{p \uparrow}(t)= & \frac{1}{2} b_{p \uparrow}(0)\left[\left(1+\frac{\epsilon_{p}}{\sqrt{\epsilon_{p}^{2}+\Delta^{2}}}\right) e^{-i t \sqrt{\epsilon_{p}^{2}+\Delta^{2}}}\right. \\
& \left.+\left(1-\frac{\epsilon_{p}}{\sqrt{\epsilon_{p}^{2}+\Delta^{2}}}\right) e^{i t \sqrt{\epsilon_{p}^{2}+\Delta^{2}}}\right]+\frac{1}{2} b_{-p \downarrow}^{\dagger}(0) \\
& \times\left[\frac{\Delta}{\sqrt{\epsilon_{p}^{2}+\Delta^{2}}} e^{-i t} \sqrt{\epsilon_{p}^{2}+\Delta^{2}}-\frac{\Delta}{\sqrt{\epsilon_{p}^{2}+\Delta^{2}}} e^{i t \sqrt{\epsilon_{p}^{2}+\Delta^{2}}}\right] .
\end{aligned}
$$

Substituting $b_{p \uparrow}(t)$ into the Green's function $\Sigma_{p} \mathbf{g}_{p}^{r}\left(t_{1}, t_{2}\right)$, taking the wide-bandwidth approximation, and assuming that $\rho_{R}^{N}(\boldsymbol{\epsilon})$ is independent with energy $\epsilon,{ }^{38}$ we then have ${ }^{23}$

$$
\begin{aligned}
\sum_{p} \mathbf{g}_{p}^{r}\left(t_{1}, t_{2}\right)_{11}= & -i \theta\left(t_{1}-t_{2}\right) \sum_{p}\left\langle\left\{b_{p \uparrow}\left(t_{1}\right), b_{p \uparrow}^{\dagger}\left(t_{2}\right)\right\}\right\rangle \\
= & -i \theta\left(t_{1}-t_{2}\right) \sum_{p}\left\{\frac{1}{2}\left(1+\frac{\epsilon_{p}}{\sqrt{\epsilon_{p}^{2}+\Delta^{2}}}\right)\right. \\
& \times e^{-i \sqrt{\epsilon_{p}^{2}+\Delta^{2}}\left(t_{1}-t_{2}\right)} \\
& \left.+\frac{1}{2}\left(1-\frac{\epsilon_{p}}{\sqrt{\epsilon_{p}^{2}+\Delta^{2}}}\right) e^{i \sqrt{\epsilon_{p}^{2}+\Delta^{2}}\left(t_{1}-t_{2}\right)}\right\}
\end{aligned}
$$

$$
\begin{aligned}
= & -i \theta\left(t_{1}-t_{2}\right) \rho_{R}^{N} \int d \epsilon_{p} \frac{1}{2}\left(e^{-i \sqrt{\epsilon_{p}^{2}+\Delta^{2}}\left(t_{1}-t_{2}\right)}\right. \\
& \left.+e^{i \sqrt{\epsilon_{p}^{2}+\Delta^{2}}\left(t_{1}-t_{2}\right)}\right) \\
= & -i \theta\left(t_{1}-t_{2}\right) \rho_{R}^{N} \int_{0}^{\infty} d \epsilon \frac{\epsilon}{\sqrt{\epsilon^{2}-\Delta^{2}}} \\
& \times\left(e^{-i \epsilon\left(t_{1}-t_{2}\right)}+e^{i \epsilon\left(t_{1}-t_{2}\right)}\right) \\
= & i \theta\left(t_{1}-t_{2}\right) \rho_{R}^{N} \int d \epsilon \frac{|\epsilon|}{\sqrt{\epsilon^{2}-\Delta^{2}}} e^{-i \epsilon\left(t_{1}-t_{2}\right)} .
\end{aligned}
$$

Notice that $p$ may have a small imaginary part from the Blonder-Tinkham-Klapnijk theory, ${ }^{40}$ so the integral over $\epsilon$ begin from 0 in Eq. (B3). Then $\Sigma_{R}^{r}\left(t_{1}, t_{2}\right)_{11}$ is easily derived under the wide-bandwidth approximation: ${ }^{38}$

$$
\begin{aligned}
\sum_{R}^{r}\left(t_{1}, t_{2}\right)_{11} & =\sum_{p} R_{p} R_{p}^{*} \mathbf{g}_{p}^{r}\left(t_{1}, t_{2}\right)_{11} \\
& =-i \Gamma_{R} \int \frac{d \epsilon}{2 \pi} \frac{\theta\left(t_{1}-t_{2}\right)|\epsilon|}{\sqrt{\epsilon^{2}-\Delta^{2}}} e^{-i \epsilon\left(t_{1}-t_{2}\right)}
\end{aligned}
$$

By using $g_{R}^{<}(\epsilon)=f_{R}(\epsilon)\left[g_{R}^{a}(\epsilon)-g_{R}^{r}(\epsilon)\right]$, the self-energy $\Sigma_{R}^{<}\left(t_{1}, t_{2}\right)$ is obtained as

$$
\Sigma_{R}^{<}\left(t_{1}, t_{2}\right)_{11}=i \Gamma_{R} \int \frac{d \epsilon}{2 \pi} e^{-i \epsilon\left(t_{1}-t_{2}\right)} f_{R}(\epsilon) \widetilde{\rho}_{R}(\epsilon)
$$

Similarly, one can obtain the other self-energy $\Sigma_{R}^{r,<}\left(t_{1}, t_{2}\right)_{\alpha \beta}$, where $\alpha, \beta=1,2$.
*Permanent address: Department of Physics, Peking University, Beijing 100871, China.

${ }^{1}$ N. S. Wingreen, Antti-Pekka Jauho, and Y. Meir, Phys. Rev. B 48, 8487 (1993); Antti-Pekka Jauho, N. S. Wingreen, and Y. Meir, ibid. 50, 5528 (1994).

${ }^{2}$ Q.-F. Sun and T.-H. Lin, Phys. Rev. B 56, 3591 (1997).

${ }^{3}$ A. Fujiwara, Y. Takahashi, and K. Murase, Phys. Rev. Lett. 78, 1532 (1997).

${ }^{4}$ L. P. Kouwenhoven et al., Phys. Rev. Lett. 73, 3443 (1994); 67, 1626 (1991).

${ }^{5}$ R. H. Blick et al., Appl. Phys. Lett. 67, 3924 (1995).

${ }^{6}$ H. Drexler et al., Appl. Phys. Lett. 67, 2816 (1995).

${ }^{7}$ B. J. Keay et al., Phys. Rev. Lett. 75, 4098 (1995); 75, 4102 (1995)

${ }^{8}$ P. K. Tien and J. P. Gordon, Phys. Rev. 129, 647 (1963).

${ }^{9}$ D. Sokolovski, Phys. Rev. B 37, 4201 (1988); H. C. Liu, ibid. 43, 12538 (1991).

${ }^{10}$ M. Wagner, Phys. Rev. Lett. 76, 4010 (1996); Q.-F. Sun, J. Wang, and T.-H. Lin, Phys. Rev. B 58, 2008 (1998).

${ }^{11}$ Y. V. Nazarov, Physica B 189, 57 (1993); C. Bruder and H. Schoeller, Phys. Rev. Lett. 72, 1076 (1994).
${ }^{12}$ P. Johansson, Phys. Rev. B 41, 9892 (1990); P. Johansson and G. Wendin, ibid. 46, 1451 (1992).

${ }^{13}$ W. R. Frensley, Phys. Rev. B 36, 1570 (1987).

${ }^{14}$ L. Y. Chen and C. S. Ting, Phys. Rev. B 43, 2097 (1991).

${ }^{15}$ Q.-F. Sun and T.-H. Lin, J. Phys.: Condens. Matter 9, 4875 (1997); 9, 3043 (1997).

${ }^{16}$ J. König, J. Schmid, H. Schoeller, and G. Schön, Phys. Rev. B 54, 16820 (1996).

${ }^{17}$ M. H. Pedersen and M. Büttiker, Phys. Rev. B 58, 12993 (1998).

${ }^{18}$ Mesoscopic Electron Transport, edited by L. L. Sohn (Kluwer, Dordrecht, 1997).

${ }^{19}$ Mesoscopic Superconductivity, edited by F. W. J. Hekking, G. Schön, and D. V. Averin [Physica B 203, 201 (1994)].

${ }^{20}$ C. J. Lambert and R. Raimondi, J. Phys.: Condens. Matter 10, 901 (1998).

${ }^{21}$ Y. Takagaki and H. Takayanagi, Phys. Rev. B 53, 14530 (1996).

${ }^{22}$ H. Takayanagi, T. Akazaki, and J. Nitta, Phys. Rev. Lett. 75, 3533 (1995).

${ }^{23}$ A. L. Yeyati, A. Martin-Rodero, and J. C. Cuevas, J. Phys.: Condens. Matter 8, 449 (1996); J. C. Cuevas, A. Martin-Rodero, and A. Levy Yeyati, Phys. Rev. B 54, 7366 (1996). 
${ }^{24}$ M. C. Koops et al., Phys. Rev. Lett. 77, 2542 (1996).

${ }^{25}$ A. W. Kleinsasser et al., Phys. Rev. Lett. 72, 1738 (1994); N. van der Post et al., ibid. 73, 2611 (1994).

${ }^{26}$ A. F. Morpurgo et al., Phys. Rev. Lett. 79, 4010 (1997).

${ }^{27}$ M. T. Tuominen et al., Phys. Rev. Lett. 69, 1997 (1992); Phys. Rev. B 47, 11599 (1993).

${ }^{28}$ T. M. Eiles, J. M. Martinis, and M. H. Devoret, Phys. Rev. Lett. 70, 1862 (1993); F. W. J. Hekking et al., ibid. 70, 4138 (1993); J. M. Hergenrother, M. T. Tuominen, and M. Tinkham, ibid. 72, 1742 (1994).

${ }^{29}$ J. M. Hergenrother et al., Phys. Rev. B 51, R9407 (1995).

${ }^{30}$ V. N. Antonov and H. Takayanagi, Phys. Rev. B 56, R8515 (1997).

${ }^{31}$ U. Hanke, M. Gisselfält, and K. A. Chao, Phys. Rev. B 54, 1529 (1996).

${ }^{32}$ Hong-Kang Zhao and G. V. Gehlen, Phys. Rev. B 58, 13660 (1998).

${ }^{33}$ C. W. J. Beenakker, Phys. Rev. B 46, 12841 (1992).
${ }^{34}$ N. R. Claughton, M. Leadbeater, and C. J. Lambert, J. Phys.: Condens. Matter 7, 8757 (1995).

${ }^{35}$ R. Fazio and R. Raimondi, Phys. Rev. Lett. 80, 2913 (1998).

${ }^{36}$ S. Ishizaka, J. Sone, and T. Ando, Phys. Rev. B 52, 8358 (1995).

${ }^{37}$ For discussions of gauge invariance, see M. Büttiker and T. Christen, in Mesoscopic Electron Transport, Vol. 345 of NATO Advanced Study Institute, Series E: Applied Science, edited by L. L. Sohn, L. P. Kouwenhoven, and G. Schoen (Kluwer Academic, Dordrecht, 1997), p. 259; Z. S. Ma, J. Wang, and H. Guo, Phys. Rev. B 57, 9108 (1998).

${ }^{38}$ N. S. Wingreen, K. W. Jacobsen, and J. W. Wilkins, Phys. Rev. B 40, 11834 (1989).

${ }^{39}$ C. A. Stafford and N. S. Wingreen, Phys. Rev. Lett. 76, 1916 (1996); Q.-F. Sun and T.-H. Lin, J. Phys.: Condens. Matter 10, 3569 (1998).

${ }^{40}$ G. E. Blonder, M. Tinkham, and T. M. Klapwijk, Phys. Rev. B 25, 4515 (1982). 